\title{
The coupled cluster method applied to quantum magnetism
}

DOI:

10.1007/BFb0119597

\section{Document Version}

Accepted author manuscript

Link to publication record in Manchester Research Explorer

\section{Citation for published version (APA):}

Farnell, DJJ., \& Bishop, RF. (2004). The coupled cluster method applied to quantum magnetism. In U. Schollwöck, J. Richter, DJJ. Farnell, \& RF. Bishop (Eds.), Quantum Magnetism (pp. 307-348). (Series on Lecture Notes in Physics; Vol. 645). Springer Nature. https://doi.org/10.1007/BFb0119597

\section{Published in:}

Quantum Magnetism

\section{Citing this paper}

Please note that where the full-text provided on Manchester Research Explorer is the Author Accepted Manuscript or Proof version this may differ from the final Published version. If citing, it is advised that you check and use the publisher's definitive version.

\section{General rights}

Copyright and moral rights for the publications made accessible in the Research Explorer are retained by the authors and/or other copyright owners and it is a condition of accessing publications that users recognise and abide by the legal requirements associated with these rights.

\section{Takedown policy}

If you believe that this document breaches copyright please refer to the University of Manchester's Takedown Procedures [http://man.ac.uk/04Y6Bo] or contact uml.scholarlycommunications@manchester.ac.uk providing relevant details, so we can investigate your claim.

\section{OPEN ACCESS}




\title{
7 The Coupled Cluster Method Applied to Quantum Magnetism
}

\author{
Damian J.J. Farnell ${ }^{1}$ and Raymond F. Bishop ${ }^{2}$ \\ 1 Unit of Ophthalmology, Department of Medicine, University Clinical \\ Departments. Daulby Street, University of Liverpool, Liverpool L69 3GA, \\ United Kingdom, d.farnelloliverpool. ac .uk \\ 2 Department of Physics, University of Manchester Institute of Science and \\ Technology (UMIST), P.O. Box 88, Manchester M60 1QD, United Kingdom, \\ r.f.bishop@umist.ac.uk
}

\begin{abstract}
The Coupled Cluster Method (CCM) is one of the most powerful and universally applied techniques of quantum many-body theory. In particular, it has been used extensively in order to investigate many types of lat tice quantum spin system at zero temperature. The ground-and excited-state properties of these systems may now be determined routinely to great accuracy. In this Chapter we present an overview of the CCM formalism and we describe how the CCM is applied in detail. We illustrate the power and versatility of the method by presenting results for four different spin models. These are, namely, the $X X Z$ model, a Heisenberg model with bonds of differing strengths on the square lattice a model which interpolates between the Kagomé- and triangular-lattice antiferromagnets, and a frustrated ferrimagnetic spin system on the square lattice. We consider the ground-state properties of all of these systems and we present accurate results for the excitation energies of the spin-half square-lattice $X X Z$ model. We utilise an "extended" SUB2 approximation scheme, and we demonstrate how this approximation may be solved exactly by using Fourier transform methods or, alternatively, by determining and solving the SUB2-m problem. We also present the results of "localised" approximation schemes called the LSUBm or SUBm-m schemes. We note that we must utilise computational techniques in order to solve these localised approximation schemes to "high order." We show that we are able to determine the positions of quantum phase transitions with much accuracy, and we demonstrate that wo are able to determine their quantum criticality by using the $\mathrm{CCM}$ in conjunction with the coherent anomaly method (CAM). Also, we illustrate that the CCM may be used in order to determine the "nodal surfaces" of lattice quantum spin systems. Finally, we show how connections to cumulant series expansions may be made by determining the perturbation series of a spin-half triangular-lattice antiferromagnet using the CCM at various levels of LSUBm approximation.
\end{abstract}

\subsection{Introduction}

Key experimental observations in fields such as superfluidity, superconductivity, nuclear structure, quantum chemistry, quantum magnetism and strongly correlated electronic systems have often implied that the strong quantum correlations inherent in these systems should be fully inchded, at least conceptually, in any theoretical calculations that aim fully to describe their basic

D.J.J. Farnell and R.F. Bishop. Ths Compled Chuser Method Applied to Quantum Magnetism, Lect. Notes Phys. 645.307 348 (2004)

http://ww.springerlink. com/

(O) Springer-Veriag Berlin Heidelberg 2004 
properties. Cntil fairly recently a common problem in many of these fields has been that the "conceptual school" of quantum many-body theory (QMBT) has been rather divorced from the "quantitative school" of fully microscopic QMBT. In this context the conceptual school typically simplifies the original. fundamental theoretical model to a more tractable one. This is done either by replacing the original Hamiltonian with a simpler or effective one that still implies or includes the most important of the observed features, and/or by postulating that these key features can be captured via an (approximate) wave function with specific inbuilt correlations. The BCS state for superconductors is a typical example of the latter. By contrast. the quantitative school attempts to solve the original quantum many-body problem as accurately as possible. Nowadays the boundaries between the two schools are becoming increasingly blured for several important reasons. Thus. on one hand many of the most interesting problems, such as high-temperature superconductivity. are so difficult that neither school can present convincing solutions. On the other hand, the techniques now available in the field of $a b$ initio QMBT have become increasingly refined over the last 15 years or so, and have also become more accessible to a wider group of researchers who can utilise the experience and expertise built up in other fields of application.

$A b$ initio techniques of microscopic QMBT are at their best. designed to include the important effects of quant um correlations in an mbiased and systematic mamner. In particular. over the last decade or so. some of the QMBT lools that have proven to be versatile in describing very accurately a wide range of both finite and extended systems of interest in physics and chemistry, and which are defined in continuous space, have begun to be applied to quantum lattice systems. They are now begiming to provide unified treatments of such systems, which can not only compete. for example, with other nuch more computationally intensive stochastic simulations, but can also provide an almost unique means to study in a systematic and unbiased manner the physically interesting (zero-temporature) quantum phase transitions that many such quantum lattice systems display in abundant variety. At the same time the conceptual school of QMBT can often provide a good starting point for the quantitative school. as we shall see in more detail below. in the form of "model" refercuce states that become the starting or zeroth-order approximations on top of which futher nany-body correlations can be systematically inchuded within well-defned herarchical approximation schemes.

Foremost among the most versatile techniques in the nodem arsenal of QMBT are those such as quantum Monte Carlo (QMC) methods $[1-4]$ the correlated basis function (CBF) method [5-15] and the coupled cluster method (CCM) [16-24], on the last of which we concentrate in this Chapter. The latter two methods are undoubtedly the most powerful and most universally applicable of all fully microscopic techniques presently available for ab initio calculations in QMBT. Each of the above methods has its own particular strengths and weaknesses, as we discuss in more detail below. Before 
doing so, however, we first give a short overview of the CBF method since, for reasons discussed more fully below, we shall concentrate our main attention hereafter on the CCM.

The most common, and perhaps the simplest, of the variational methods in QMBT are based on trial wave functions of the (Bijl-Dingle-) Jastrow form [25]. Early calculations of this sort relied on various cluster expansions of the ensuing approximate matrix elements [25-27]. It was realised later that these variational approaches may also be formulated diagrammatically [28]. This feature has been of considerable help in the construction of such powerful approximations as the Percus-Yevick and hypernetted chain (HNC) summations and their variants, which have their origins in the classical theory of liquids and which have been adapted for both bosonic and fermionic systems [7.29]. The review article by Clark [8] gives a good overview of the variational theory sketched above as applied to extended nuclear matter. The interested reader is also referred to [30].

Two basic flaws mar the above variational approaches. Firstly, the particular partial summations of the graphs considered by such approximations as the HNC approach destroy one of the most attractive features of variational techniques, namely that they yield upper bounds to the exact ground-state cnergy. Secondly, even a complete summation of graphs (or a variational Monte Carlo evaluation of the corresponding expectation values) for a given trial wave function (of Jastrow type, for example) gives only the exact variational result and not the true ground state. This latter deficiency may be remedied by the inclusion of more general state-dependent correlations and higher-order correlation functions of the Feenberg type. Alternatively, and more generally, one may extend the Jastrow wave function to a complete set of correlated basis functions, which is the CBF approach.

The CBF method was introduced some 45 years ago by Feenberg and his collaborators [5-7], and was later developed largely by Clark and his collaborators [8-11]. Introductory surveys of the method are given in [12-15]. We simply note here that the CBF method has as its central ingredient the direct incorporation of the most important interparticle correlations into the approximate wave functions on which the microscopic description is based. At its simplest level the method only involves a single configuration, and hence reduces to ordinary variational theory. This further reduces to Jastrow theory if the simplest reasonable choice of correlation operator is made in terms of the usual symmetric product over all pairs in the system of state-independent two-body correlation functions.

Since we shall be dealing extensively with applications of the $\mathrm{CCM}$ in this Chapter, we postpone a comparable introduction of it until Sec. 1.2, and before doing so we retumn to a review of the relative merits and weaknesses of the QMC, CBF and CCM approaches to QMBT. We first note that QNC techniques are severely restricted in the choice of problems to which they can readily be applied by the infamous "sign problem" $[31,32]$, which arises whenever we bave a lack of prior knowledge of the nodal surface of the many- 
body wave function under discussion. For spin systems on a regular lattice it is often related to the occurrence of (strong) frustration. Conversely it can only readily be circumvented when we have such prior knowledge via. for example, the Marshall-Peierls sign rule [33]. or some such andogous relation. Nevertheless. QMC numerical results for spin-lattice systems often provide the benchmark for other methods for the cases in which the technique can be applied. especially for lattices in two or more spatial dimensions. We note in passing that for the special case of one spatial dimension (i.e. chains) the methods of choice usually inchue exact solutions when available [34 37]. the density matrix renomalisation group (DNRG) method [38]. and techniques from quantun field theory [39].

By contrast. The CBF method is not limited in the range of systems to which it can be applied by the presence of strong (geometric or dynamic) frustration. However, its applications up till now have been restricted in practice to a very limited number of spin-lattice systems (namely, the transverse Ising model [40-43] and the $X Y$ model [44]. We note that part of the reason for this limited nsage of the method for problems in quantum magnetism lies in the fact that. in practical calculations. it is often difficult to include correlations beyond the two-body level in the Jastrow-Feenberg trial states. Such higher-order correlations are often important for very accurate calculations.

By further contrast. the CCN is limited noither by the presence of frustration in the system nor to the inclusion of only two-body correlations. As we shall see later. the inclusion of mamy-body correlations between spins up to about the 8-body level or so is nowadays quite routine. It is important to note that the Goldstone linked-chnster theorem is explicitly obeyed by the C CM at any level of approximate implementation. and hence results may always be determined directly from the outset in the infinite lattice limit. $N \rightarrow x$ (where $N$ is the number of spins in the system). This is in sharp contrast to the QNC results that are always obtained for finite-sized lattices from which the jesults for the infinite lattice need to bo extrapolated using finitesize scaling arguments. Furthermore. the very important Hellmann-Feyman theorem is also obeyed by the C CN at all levels of approximation. On the other hand. we note that in order to retain all of these useful and important features. it tums out to be necessary to relax the condition that the corresponding bra- and ket-states are manifestly Hermitian conjugates of one another. At a given level of trmeation. this Hermiticity property may be only approximately obeyed. although it is certainly restored in the cxact limit. As we shall see a consequence is that we lose the property in the CCM that the results for the ground-state energy form an upper bound to the true results. In practice this lack of manifest Herniticity poses few actual problems. Indeed. it can often be used as an internal quality check on the accuracy of the method. Finally. we note that the CCM lends itself extremely woll for applications on the latice to the use of computer-algebraic techiques both to derive and to solve the fundamental sets of coupled nomlinear 
equations that lie at its heart in practical implementations, via well-defined hierarchies of approximations.

In the rest of this Chapter we will focus attention only on spin-lattice applications of the CCM, for reasons already cited. Nevertheless, we believe that the CBF method still has a worthy future in this field. We hope that others will still develop it further, since it certainly shares many highly desirable features with the CCM. Before concentrating in the rest of this Chapter solely on the CCM, we take a final opportunity to list some of the more important of these features below:

- Both methods are extremely versatile, and they have been extensively tested. There is by now a large amount of experience in using them.

- An impressively wide range of applications to systems of physical interest has been made of one and/or the other method. These include finite nuclei; nuclear matter; quantum field theory (including systems of anharmonic oscillators, $\phi^{4}$ field theory, and pion-mucleon field theory); atoms and molecules of interest in quantum chemistry; the electron gas; quantum hydrodynamics; and the liquids helium (including bulk ${ }^{3} \mathrm{He}$ and ${ }^{4} \mathrm{He}$ and their mixtures, and films).

- Both methods are capable of very high accuracy at attainable levels of implementation. In most applications the CBF and/or CCM results are either the best or among the best from all available microscopic techniques. They are now often at the point of being fully competitive with the large-scale QMC simulations in the cases where the latter can be performed.

- Neither method is restricted in principle to particular forms of the Hamiltonian. Both are easily capable of handling very complicated interactions.

- Both the CBF method and the CCM are intrinsically nonperturbative in nature. Some correlations are retained to infinite order, even at the lowest levels of implementation. The CCM, in particular, can often be used to derive (or reconstruct) perturbation theory (PT) series, by a suitable choice of truncation hierarchy for the subsets of terms retained in the multiconfigurational expansions of the ground-or excited-state correlation operators, as described more fully below. In such cases, the CCM provides a natural analytic contimuation of the $\mathrm{PT}$ series, which in practice is usually found to be valid far outside the radius of convergence of the PT series, and also to be quantitatively superior to such alternative schemes as (generalised) Padé resummations.

- Although nonperturbative in principle, the CCM can be easily related to the Goldstone diagram expansions of time-independent perturbation theory. This feature facilitates comparisons with other methods.

- Similarly, at the optimised Jastrow level implemented via the HNC approximation, the CBF method has been shown [45] to be equivalent to two-body localised parquet theory, and hence to a sum of planar Feymman diagrams of time-dependent perturbation theory. 
- In both methods one may work from the outset in the bulk thermodynamic limit. $N \rightarrow \infty$. thereby avoiding problems connected with finite-size effects. This is always done in the CCM. although. for technical reasons, it is not always practicable in the CBF rase.

- Both methods have the virtue of great flexibility. One may choose "uncorrelated" or "model" ground-state reference states. for example, in many ways. In particular. this presents an opportunity for the "conceptual school" of many-body theory to provide a good starting-point for the "quantitative school." Similarly, many different approximation hierarchies for the correlation operators of the CBF and $\mathrm{CCN}$ schemes can be envisaged. and there is again room for external experience or physical intuition to be utilised in their choice.

- Both methods are capable of handling phase transitions. Even when the "uncorrelated" or "model" reference state is a poor choice, both the CBF and CCM sehemes have been shown in particular cases to be able to predict phase changes. In the case of the CCM we discuss this in more detail below.

- Both methods, but particulary the CCM, often have the practical capability of implementation to high orders of approximation. The CCM has especially been shown to be very amenable to the use of computer algebra to derive the high-order basic coupled sets of nonlinear equations that underpin it. This feature is particularly marked for lattice systems, and it is a key reason why the CCM is now proving to be fully competitive with large-scale QNIC stochastic simulations at a fraction of the computing cost. in those cases where the latter can be performed.

For further details of the $\mathrm{CBF}$ method and some of its applications to various quantum lattice systems, the interested reader is referred to the overview in [46]. where comparisons are also made with the $\mathrm{CCM}$.

Henceforth we confine our attent ion to the CCM. whose applications over the last ten or so years to quantum magnetic systems at zero temperature [47-68] have proven to be extremely successful. In particular, the use of computer-algebraic implementations of the CCM for quantum systems of large or infinite numbers of particles has largely been pioneered with respect to these spin-lattice problems. We note too in this context that there have been subsequent applications of these highly accurate computational CCA techniques to other types of lattice quantum systems. such as $U(1)$ and SU(N) lattice gauge field theory [69-71], and the latticised $O(N)$ nonlinear sigma model of relevance to chiral meson field theory [72].

In the remainder of this Chapter we firstly give a brief description of the CCM formalism. We then describe four specific applications of the method to various spin-lattice systems at zero temperature. The first application is to the unfrustrated spin-half $X X Z$ model (or anisotropic Heisenberg model) on the linear chain and on the bipartite square lattice. This simple model serves both to illustrate how the method may be applied in practice and 
to indicate the quality of the results attainable at practical levels of implementation. By contrast to this simple model, frustrated systems generally are both more difficult to deal with and have richer phase diagrams, which contain phases of novel forms of order. Three such strongly frustrated systems are then considered. The first of these, the so-called $J-J^{\prime}$ model, is a spin-half Heisenberg model on the square lattice with two different, competing nearest- neighbour couplings with different bond strengths arranged in a regular zigzag pattern. For the case where the bond strengths have different signs the square plaquettes are thus dynamically frustrated, whereas when the bond strengths have the same sign the model exhibits competition (between magnetic order and dimerisation) without frustration. The third model exhibits geometric frustration, and is again a spin-half Heisenberg model that interpolates smoothly between a triangular lattice and a Kagomé lattice. The last model considered is another model that includes the possibility of dynamical frustration, in which we have both nearest-neighbour and next-nearest-neighbour Heisenberg interactions with unequal strengths. Furthermore, the model is taken to represent a spin-half/spin-one ferrimagnet in which one sublattice of the bipartite square lattice is populated entirely with spin-one spins, while the other sublattice is populated entirely with spin-half spins. The Chapter is concluded with a diseussion of the implications of these illustrative results for further work, and with some ideas for future extensions and applications of the CCM.

\subsection{The CCM Formalism}

A brief description of the normal coupled cluster method (NCCM) formalism is now provided, although the interested reader is referred to [16--24,47-68]. for further details. The exact ket and bra ground-state energy eigenvectors, $|\Psi\rangle$ and $\langle\tilde{\Psi}|$, of a general many-body system described by a Hamiltonian $H$,

$$
H|\Psi\rangle=E_{g}|\Psi\rangle ; \quad\langle\tilde{\Psi}| H=E_{g}\langle\tilde{\Psi}|,
$$

are parametrised within the single-reference CCM as follows:

$$
\begin{gathered}
|\Psi\rangle=\mathrm{e}^{S}|\Phi\rangle ; \quad S=\sum_{I \neq 0} \mathcal{S}_{I} C_{I}^{+} \\
\langle\tilde{\Psi}|=\langle\Phi| \tilde{S} \mathrm{e}^{-S} ; \quad \tilde{S}=1+\sum_{I \neq 0} \tilde{S}_{I} C_{I}^{-}
\end{gathered}
$$

The single model or reference state $|\Phi\rangle$ is required to have the property of being a cyclic vector with respect to two well-defined Abelian subalgebras of multi-configurational creation operators $\left\{C_{j}^{+}\right\}$and their Hermitian-adjoint destruction counterparts $\left\{C_{I}^{\cdots} \equiv\left(C_{j}^{+}\right)^{\dagger}\right\}$. Thus, $|\Phi\rangle$ plays the role of a vacuum state with respect to a suitable set of (mutually commuting) many-body creation operators $\left\{C_{I}^{+}\right\}$. Note that $C_{I}^{-}|\Phi\rangle=0, \forall I \neq 0$. and that $C_{0}^{-} \equiv 1$, the 
identity operator. These operators are furthermore complete in the manybody Hilbert (or Fock) space. Also, the correlation operator $S$ is decomposed entirely in terms of these creation operators $\left\{C_{I}^{+}\right\}$, which. when acting on the model state $\left(\left\{C_{1}^{+}|\Phi\rangle\right\}\right)$, create excitations from it. We note that although the manifest Hermiticity. $(\langle\tilde{\Psi}|=| \Psi\rangle /\langle\Psi \mid \Psi\rangle)$. is lost the normalisation conditions $\langle\tilde{\psi} \mid \Psi\rangle=\langle\Phi \mid \psi\rangle=\langle\Phi \mid \Phi\rangle \equiv 1$ are explicitly imposed. The correlation coefficients $\left\{\mathcal{S}_{1} . \mathcal{S}_{1}\right\}$ are regarded as being independent variables, and the full set $\left\{\mathcal{S}_{I}, \tilde{S}_{I}\right\}$ thus provides a complete description of the ground state. For instance, an arbitrary operator $A$ will have a ground-state expectation value given as.

$$
A \equiv\langle\tilde{\psi}|A| \Psi\rangle=\left\langle\Phi\left|\tilde{S}^{-S} A e^{S}\right| \Phi\right\rangle=\bar{A}\left(\left\{\mathcal{S}_{I}, \tilde{S}_{I}\right\}\right)
$$

We note that the exponentiated form of the ground-state CCM parametrisation of (7.2) ensures the correct counting of the independent and excited correlated many-body clusters with respect to $|\Phi\rangle$ which are present in the exact ground state $|\Psi\rangle$. It also ensures the exact incorporation of the Goldstone linked-cluster theorem. which itself guarantees the size-extensivity of all relevant extensive physical quantities. We also note that any operator in a similarity transform may be written as

$$
A=e^{-S} A e^{S}=A+[A, S]+\frac{1}{2 !}[[A, S], S]+\cdots
$$

The determination of the correlation coefficients $\left\{\mathcal{S}_{I}, \tilde{\mathcal{S}}_{I}\right\}$ is achieved by taking appropriate projections onto the ground-state Schrödinger equations of (7.1). Equivalently, they may be determined variationally by requiring the ground-state energy expectation functional $\vec{H}\left(\left\{\mathcal{S}_{I}, \tilde{\mathcal{S}}_{I}\right\}\right)$, defined as in (7.3). to be stationary with respect to variations in each of the (independent) variables of the full set. We thereby easily derive the following coupled set of equations.

$$
\begin{aligned}
& \delta H / \delta \tilde{\mathcal{S}}_{I}=0 \Rightarrow\left\langle\Phi\left|C_{I}^{-} \mathrm{e}^{-S} H \mathrm{e}^{S}\right| \Phi\right\rangle=0, \forall I \neq 0 ; \\
& \delta H / \delta \mathcal{S}_{I}=0 \Rightarrow\left\langle\Phi\left|\tilde{S e}^{-S}\left[H . C_{I}^{+}\right] \mathrm{e}^{S}\right| \Phi\right\rangle=0, \forall I \neq 0 .
\end{aligned}
$$

Equation (7.5) also shows that the ground-state cnergy at the stationary point has the simple form

$$
E_{g}=E_{g}\left(\left\{S_{l}\right\}\right)=\left\langle\Phi\left|e^{-S} H e^{S}\right| \Phi\right\rangle
$$

It is important to realize that this (bi-) variational formulation does not lead to an upper bound for $E_{g}$ when the summations for $S$ and $S$ in $(7.2)$ are truncated, due to the lack of exact Hermiticity when such approximations are made. However, one can prove that the important Helmann-Feyman theorem is preserved in all such approximations. 
We note that (7.5) represents a coupled set of non-linear multinomial equations for the c-number correlation coefficients $\left\{\mathcal{S}_{I}\right\}$. The nested commutator expansion of the similarity-transformed Hamiltonian

$$
\tilde{H} \equiv e^{-S} H e^{S}=H+[H, S]+\frac{1}{2 !}[[H, S], S]+\cdots
$$

and the fact that all of the individual components of $S$ in the sum in $(7.2)$ commute with one another, together imply that each element of $S$ in $(7.2)$ is linked directly to the Hamiltonian in each of the terms in (7.8). Thus, each of the coupled equations (7.5) is of linked cluster type. Furthermore, each of these equations is of finite length when expanded, since the otherwise infinite series of (7.8) will always terminate at a finite order, provided only (as is usually the case) that each term in the second-quantised form of the Hamiltonian. $H$, contains a finite number of single-body destruction operators. defined with respect to the reference (vacumm) state $|\Phi\rangle$. Hence the CCM parametrisation naturally leads to a workable scheme which can be efficiently implemented computationally. It is important to note that at the heart of the CCM lies a similarity transformation, in contrast with the unitary transformation in a standard variational formulation in which the bra state $\langle\tilde{\psi}|$ is simply taken as the explicit Hermitian conjugate of $|\Psi\rangle$.

In the case of spin-lattice problems of the type considered here, the operators $C_{l}^{+}$become products of spin-raising operators $s_{k}^{+}$over a set of sites $\{k\}$, with respect to a model state $|\Phi\rangle$ in which all spins points "downward" in some suitably chosen local spin axes. The CCM formalism is exact in the limit of inclusion of all possible such multi-spin cluster correlations for $S$ and $\tilde{S}$, although in any real application this is usually impossible to achieve. It is therefore necessary to utilise various approximation schemes within $S$ and $\tilde{S}$. The three most commonly employed schemes previously utilised have been: (1) the SUB $n$ scheme, in which all correlations involving only $n$ or fower spins are retained, but no further restriction is made concerning their spatial separation on the lattice; (2) the SUBn- $m$ sub-approximation, in which all SUBn correlations spanning a range of no more than $m$ adjacent lattice sites are retained; and (3) the localised LSUBm scheme, in which all multi spin correlations over all distinct locales on the lattice defined by m or fewer contiguous sites are retained.

An excited-state wave function, $\left|\Psi_{\uparrow}\right\rangle$, is determined by linearly applying an excitation operator $X^{t}$ to the ket-state wave function of (7.2), such that

$$
\left|\Psi_{c}\right\rangle=X^{e} e^{s}|\Phi\rangle
$$

This equation may now be used to determine the low-lying excitation energies, such that the Schrödinger equation, $H\left|\Psi_{e}\right\rangle=E_{e}\left|\Psi_{e}\right\rangle$, may be combined with its ground-state counterpart of (7.1) to give the result,

$$
\epsilon_{e} X^{e}|\Phi\rangle=e^{-S}\left[H, X^{e}\right] e^{S}|\Phi\rangle
$$


where $\epsilon_{t:} \equiv E_{e}-E_{g}$ is the excitation energy. By analogy with the ground-state formalism, the excited-state correlation operator is written as,

$$
X^{r}=\sum_{I \neq 0} \mathcal{X}_{I}^{\prime} C_{I}^{+}
$$

where the set $\left\{C_{I}^{+}\right\}$of multi-spin creation operators may differ from those used in the ground-state parametrisation in (7.2) if the excited state has different quantum numbers than the ground state. We note that $(7.11)$ implies the overlap relation $\left\langle\Phi \mid \Psi_{c}\right\rangle=0$. By applying $\langle\Phi| C_{y}^{-}$to $(7.10)$ we find that,

$$
\epsilon_{t} \mathcal{X}_{I}^{\prime}=\left\langle\Phi\left|C_{I}^{-} e^{-S^{S}}\left[H . X^{\prime}\right] e^{S}\right| \Phi\right\rangle, \forall I \neq 0
$$

which is a generalised set of eigenvalue equations with eigenvalues $\epsilon_{\epsilon}$ and corresponding eigenvectors $\mathcal{X}_{J}^{\prime}$, for each of the excited states which satisfy $\left\langle\Phi \mid \Psi_{c}\right\rangle=0$

We note that lower orders of approximation may be determined andytically and an example of applying the LSUB2 and SUB2 approxinations to the spin-half linear chain $X X Z$ nodel is given later in order to show clearly how this is performed. However, it rapidly becomes clear that analytical determination of the CCN equations for higher orders of approximation is impractical. We therefore employ computer algebraic techniques in order efficiently to determine and solve the CCM ket-and bra-state equations. A full exposition of this topic is beyond the scope of this chapter. although we note that the problem essentially becomes one of pattern matching in order to determine the CCM ground-state ket equations. The bra-state equations may be determined easily thereafter and the ket-and bra-state equations are readily solved using standard techniques for the solution of coupled polynomial equations (e.g., the Newton-Raphson method). The excited-state eigenvalue equations may be also detemined in an analogous manner, and. although this is not strictly necessary, we restrict the level of approximation to the same for the excited state as for the ground state in calculations presented here. A full exposition of the details in applying the CCM to high orders of approximation is given for the ground state in [54.59.67] and for excited states in [62].

Note that the results of SCBm-m and LSCBm approximation schemes may be extrapolated to the exact limit, $m \rightarrow \infty$. using various "heuristic" approaches. How to do this is not discussed here, although the interested reader is referred to [59.67] for more details.

\subsection{The $X X Z$ Model}

We wish to apply the CCM to the spin-half $X X Z$ model on the linear chain and the square lattice in order to illustrate how one applies the CCM to a practical problem and also to demonstrate the accuracy and power of the 
method. We note that these systems are unfrustrated and, in global spin coordinates, the $X X Z$ Hamiltonian is specified as follows,

$$
H=\sum_{\langle i, j\rangle}\left[s_{i}^{x} s_{j}^{x}+s_{i}^{y} s_{j}^{y}+\Delta s_{i}^{z} s_{j}^{z}\right] .
$$

where the sum on $\langle i, j\rangle$ counts all nearest-neighbour pairs once. The Néel state is the ground state in the trivial Ising limit $\Delta \rightarrow \infty$, and a phase transition occurs at (or near to) $\Delta=1$. Indeed, the ground state demonstrates Néellike order in the $z$-direction for $\Delta>1$ and a similar $x-y$ planar phase for $-1<\Delta<1$. The system is ferromagnetic for $\Delta<-1$.

\subsubsection{The CCM Applied to the $X X Z$ Model Using a $z$-Aligned Néel Model State}

We turn now to the choice of $|\Phi\rangle$ and the operators $\left\{C_{I}^{+}\right\}$for the case of spin-half quantum antiferromagnets on bipartite lattices, in regimes where the corresponding classical limit is described by a Néel-like order in which all spins on each sublattice are separately aligned in some global spin axes. It is then convenient to introduce a different local quantisation axis and different spin coordinates on each sublattice, by a suitable rotation in spin space, so that the corresponding reference state becomes a fully aligned ("ferromagnetic") state, with all spins pointing along, say, the negative z-axis in the corresponding local axes. Such rotations are cannonical tranformations that leave the spin commutation relations unchanged. In the same local axes, the configuration indices $I \rightarrow\left\{k_{1}, k_{2}, \cdots, k_{M}\right\}$, a set of site indices, such that $C_{I}^{+} \rightarrow s_{k_{1}}^{+} s_{k_{2}}^{+}$. $\cdots s_{k_{M}}^{+}$, where $s_{k}^{ \pm} \equiv s_{k}^{x} \pm i s_{k}^{y}$ are the usual spin-raising and spin-lowering operators at site $k$.

For the Hamiltonian of (7.13) we first choose the $z$-aligned Néel state as our reference state (which is the exact ground state for $\Delta \rightarrow \infty$, and is expected to be a good starting point for all $\Delta>1$, down to the expected phase transition at $\Delta=1$ ). We then perform a rotation of the up-pointing spins by $180^{\circ}$ about the $y$-axis, such that $s^{x} \rightarrow-s^{x}, s^{y} \rightarrow s^{y}, s^{z} \rightarrow-s^{z}$ on this sublattice. The Hamiltonian of (7.13) may thus be written in these local coordinates as.

$$
H=-\frac{1}{2} \sum_{<i, j>}\left[s_{i}^{+} s_{j}^{+}+s_{i}^{-} s_{j}^{-}+2 \Delta s_{i}^{z} s_{j}^{z}\right] .
$$

The results presented below are based on the SUB2 approximation scheme and the localised LSUBm scheme, in which we include all multispin correlations over all possible distinct locales (or "lattice animals") on the lattice defined by $m$ or fewer contiguous sites. We include all fundamental configurations, $I \rightarrow\left\{k_{1}, k_{2}, \cdots k_{n}\right\}$, with $n \leq m$, which are distinct under the point and space group symmetries of both the lattice and the Hamiltonian. The 
numbers. $N_{F}$ and $N_{F_{1}}$. of such fundamental configurations for the ground and excited states, respectively, may be further restricted by the use of additional conservation laws. For example. the Hamiltonian of (7.13) commutes with the total uniform magnetisation. $s \frac{\tilde{T}}{T}=\sum_{k} s_{k}^{z}$, where the sum on $k$ rms over all lattice sites. The gromul state is known to he in the $s_{\%}=0$ subspace. and henee we exclude configurations with an odd number of spins or with nequal numbers of spins on the two equivalent sublattices. Similarly for the excited states. sine we are only interested in the lowest-lying excitation, we restrict the choice of configurations to those with $s^{2}= \pm 1$.

\subsubsection{The LSUB2 Approximation for the Spin-Half, Linear-Chain $X X Z$ Model}

We start the LSUB2 calculation by specifying the commutation relations $\left[s_{l}^{ \pm}, s_{h}^{z}\right]=\mp s_{k}^{t} \delta_{l, k}$ and $\left[s_{l}^{+}, s_{k}^{-}\right]=2 s_{k}^{z} \delta_{l, k}$. We again note that the similarity transform may be expanded ats a series of nested commutators in (7.4). We write the LSUB2 ket-state operator in the following simple form for the spinhalf linear chain model.

$$
S=b_{1} \sum_{i}^{N} s_{i}^{+} s_{i+1}^{+}
$$

where $i$ runs over all sites on the linear chain and $b_{1}$ is the sole ket-state correlation coefficient. In this approximation we may therefore determine similarity transformed versions of the spin operators, given by

$$
\begin{aligned}
& \bar{s}_{l}^{+}=s_{i}^{+} \\
& \bar{s}_{i}=s_{i}^{\bar{r}}+b_{1}\left(s_{l}^{+} s_{l+1}^{+}+s_{l-1}^{+} s_{l}^{+}\right) \\
& \tilde{s}_{l}=s_{l}^{-}-2 b_{1}\left(s_{i}^{+} s_{l+1}^{+}+s_{l-1}^{+} s_{i}^{\tilde{l}}\right)-2 b_{l}^{2} s_{l-1}^{+} s_{l}^{+} s_{l+1}^{+}
\end{aligned}
$$

We note that the otherwise infinite-series of operators in the expansion of the similarity transform terminates to finite order. We also note that $\left(s_{l}^{+}\right)^{2}|\Phi\rangle=$ 0 for any lattice site (which is true only for spin-half systems), and this is implicitly assumed in the last of (7.16). Clearly we may also write the similarity transformed version of the Hamiltonian as

$$
\ddot{H}=-\frac{1}{2} \sum_{\langle i . j\rangle}\left[\tilde{s}_{i}^{+} \tilde{s}_{j}^{+}+\tilde{s}_{i}^{-} \tilde{s}_{j}^{-}+2 \Delta \tilde{s}_{i}^{z} \tilde{s}_{j}^{z}\right]
$$

We may now substitute the expressions for the spin operators in (7.16) into the above expression. The ground-state energy is given by

$$
\frac{E_{g}}{N}=-\frac{1}{4}\left\{\Delta+2 b_{1}\right\}
$$


We note that our expression for the ground-state energy is size-extensive (i.e, it scales linearly with $N$ ), as required by the Goldstone theorem which is obeyed by the NCCM. Furthermore, this expression terminates to finite order, as for the similarity transformed versions of spin operators. Finally, we note that any other non-trivial choice for $S$ will always yield this expression for the ground-state energy. The task is now to find $b_{1}$ and we note that if we could include all possible spin correlations in $S$ then we would obtain an exact result for the ground-state energy. However, this is found to be impossible to achieve for most cases in practice, and we make an approximation (such as the LSUB2 approximation presented here). The LSUB2 ket-state equation is given by

$$
3 b_{1}^{2}+2 \Delta b_{1}-1=0,
$$

which therefore implies that the LSUB2 ground-state energy may be written explicitly in terms of $\Delta$ as,

$$
\frac{E_{g}}{N}=-\frac{\Delta}{12}-\frac{1}{6} \sqrt{\Delta^{2}+3}
$$

We note that this expression gives the correct result in the Ising limit $\Delta \rightarrow \infty$. We again note that the bra state does not manifestly have to be the Hermitian conjugate of the ket state, and we note that the bra-state correlation operator for the LSUB2 approximation is given by,

$$
\tilde{S}=1+\tilde{b}_{1} \sum_{j}^{N} s_{j}^{-} s_{j+1}^{-}
$$

where the index $j$ runs over all sites on the linear chain and $\dot{b}_{1}$ is the sole bra-state correlation coefficient in the LSUB2 approximation. In order to determine the bra-state equation, we now explicitly determine $\bar{H} /\left(\left\{\mathcal{S}_{l}, \overline{\mathcal{S}}_{I}\right\}\right)$.

$$
\bar{H}=-\frac{N}{4}\left(\Delta+2 b_{1}\right)+N \tilde{b}_{1}\left(-\frac{1}{2}+\Delta b_{1}+\frac{3}{2} b_{1}^{2}\right) .
$$

such that LSUB2 bra-state equation is given from $\partial \bar{H} \partial b_{1}=0$ as

$$
-\frac{1}{2}+\Delta \tilde{b}_{1}+3 b_{1} \tilde{b}_{1}=0
$$

which gives $\tilde{b}_{1}=\frac{1}{2}\left(\Delta^{2}+3\right)^{-1 / 2}$. Finally, we note that once the values for the bra- and ket-state correlation coefficients have been determined (at a given level of approximation) then we may also obtain the values for expectation values, such as the sublattice magnetisation given by

$$
M \equiv-\frac{2}{N}\left\langle\tilde{\Psi}\left|\sum_{i}^{N} s_{i}^{z}\right| \Psi\right\rangle=-\frac{2}{N}\left\langle\Phi\left|\tilde{S} e^{-S}\left(\sum_{i}^{N} s_{i}^{z}\right) e^{S}\right| \Phi\right\rangle
$$


The sublattice magnetisation is written here in terms of the "rotated" spin coordinates. We note that this is given by

$$
\begin{aligned}
M_{\mathrm{LST}: 32} & =1-4 b_{1} \tilde{b}_{1} . \\
& =\frac{1}{3}\left[1+\frac{2 \Delta}{\sqrt{\Delta^{2}+3}}\right]
\end{aligned}
$$

for the LSUB2 approximation.

\subsubsection{The SUB2 Approximation for the Spin-Half, Linear-Chain $X X Z$ Model}

The SUB2 approximation allows 1 s to include all possible two-spin correlations in our wave function. We note that the SUB2 ket-state operator is given by

$$
S=1 / 2 \sum_{i}^{N} \sum_{i} b_{r} s_{i}^{+} s_{i+r}^{+}
$$

and that the index $i$ runs over all sites on the linear chain. Furthermore, the index $r$ runs over all lattice vectors which connect one sublattice to the other and $b_{r}$ is its corresponding SUB2 ket-state correlation coefficient for this vector. We again determine a similarity transformed version of the spin operators and we are able to determine the SUB2 equations, given by

$$
\sum_{p}\left\{\left(1+2 \Delta b_{1}+2 b_{1}^{2}\right) \delta_{p . r}-2\left(\Delta+2 b_{1}\right) b_{r}+\sum_{s} b_{r+s+\rho} b_{s ;}\right\}=0
$$

where $\rho$ rums over all (1D) nearest-neighbour lattice vectors. Equation (7.27) may now be solved by employing a sublattice Fourier transform. given by

$$
I(a)=\sum_{r} e^{\mathrm{i} r^{\prime}} b_{r} .
$$

where $r$ again is a lattice vector (i.e. an odd integer number in $1 D$ ) which connects the different sublattices. This expression has an inverse given by

$$
b_{r}=\int_{0}^{\pi} \frac{d q}{\pi} \cos (r q) \Gamma(q)
$$

The SUB2 equations (7.27) and (7.28) therefore lead to an expression for $\Gamma(q)$ given by

$$
\Gamma(q)=\frac{h}{\cos (q)}\left[1 \pm \sqrt{\left.1-k^{2} \cos ^{2}(q)\right]}\right.
$$

where $K=\Delta+2 b_{1}$ and $k^{2}=\left(1+2 \Delta b_{1}+2 b_{1}^{2}\right) / K^{2}$. (Note that we choose the negative solution in $(7.30)$ in order to reproduce results in the trivial limit 
$\Delta \rightarrow \infty$.) These equations now yield a self-consistency requirement on the variable $b_{1}$ and they may be solved iteratively at a given value of $\Delta$. Indeed. we know that all correlation coefficients must tend to zero (namely, for SUB2: $\left.b_{r} \rightarrow 0, \forall r\right)$ as $\Delta \rightarrow \infty$ and we track this solution for large $\Delta$ by reducing $\Delta$ in small successive steps. We find that the discriminant in (7.30) becomes negative at a critical points, $\Delta_{6} \approx 0.3728$. Furthermore, the behaviour of $b_{r}$ changes from exponential to algebraic decay with respect to $r$ at $\Delta_{c}$. These are strong indications that the CCM critical point is detecting the known quantum phase transition in the system at $\Delta=1$. Furthermore, the SUB2 approximation for the ground state may be used in conjunction with a SUB1 approximation for the excited state operator $X^{*}$ in (7.11) in order to determine the excitation spectrum. We note that the excitation spectrum becomes soft at the critical point, $\Delta_{c}$. This is further evidence for a phase transition and the interested reader is referred to [48] for more details.

We may also solve the SUB2-m equations directly using computational techniques. Indeed, we study the limit points of these approximations by using solution-tracking software (PITCON), which allows one to solve coupled nonlinear equations. We again track our solution from the limit $\Delta \rightarrow \infty$ down to and beyond the limit point and Fig. 7.1 shows our results. In particular, we note that we have two distinct branches, although only the upper branch is a "physical" solution. We again note that the CCM does not necessarily always provide an upper bound on the ground-state energy $\cdots$ although this is often the case for the physical solution! By tracking from a point at which we are sure of, the solution we guarantee that our solution is valid, and this approach is also used for LSUBM approximations.

We find that the two branches collapse onto the same line, namely, that of the full SUB2 solution, as we increase the level of SUB2-m approximation with respect to $m$. Indeed, we may plot the positions of the SUB2-m limit points against $1 / \mathrm{m}^{2}$ and we note that these data points are found to be both highly linear and they tend to the critical value, $\Delta_{c}$, for the full SUB2 equations in the limit $m \rightarrow \infty$. Again, we note that the LSUB $m$ and SUB $m-m$ approximations also show similar branches (namely, one "physical" and one "unphysical" branch) which appear to converge as one increases the magnitude of the truncation index, $m$. This is a strong indication that our LSUBm and $S U B m-m$ critical points are also reflections of phase transitions in the real system and that our extrapolated $\mathrm{LSUB} m$ and $\mathrm{SUB} m-m$ results should tend to the exact solution.

\subsubsection{CCM Results for the Spin-Half Square-Lattice $X X Z$ Model Using a $z$-Aligned Model State}

We shall now illustrate the power and accuracy of the CCM by presenting results in Figs. 7.27 .4 respectively for the energy per spin $\left(E_{g} / N\right)$ and the sublattice magnetisation $(M)$ for the ground state, and the energy gap $\left(\epsilon_{0}\right)$ 


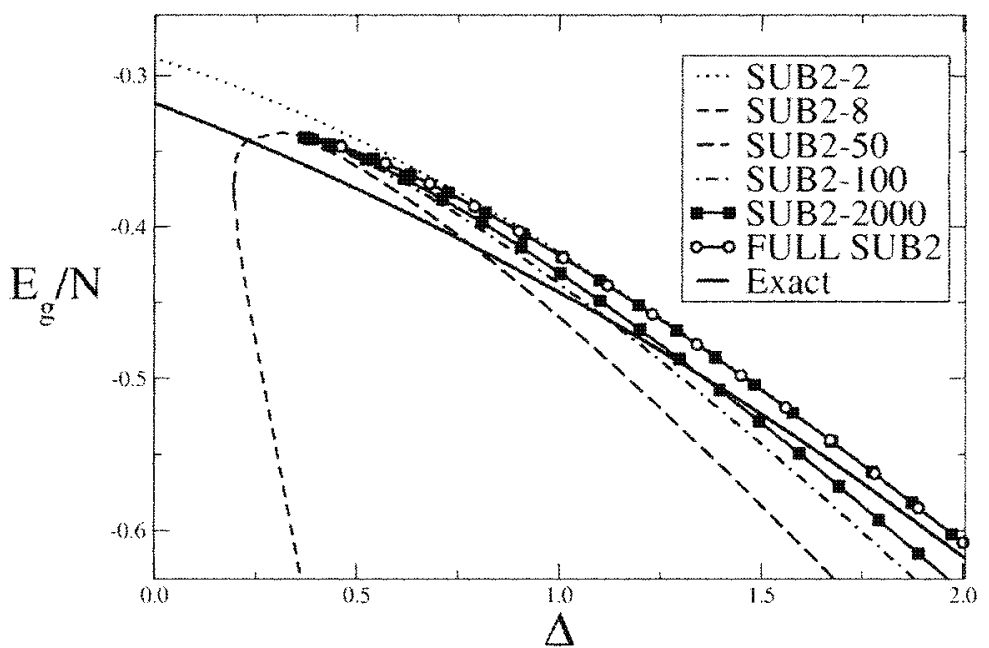

Fig. 7.1. CCM SUB2-m and full SUB2 results for the ground-state energy of the spin-half linear-chain $X X Z$ model compared to exact results of the Bethe Ansatz [34-37]. The CCM model state is the z-aligned Neel state. SUB2-m limit points converge to the SUB2 limit at $\Delta_{c}=0.3728$, at which point the solution to the full SUB2 equations becomes complex. as $m \rightarrow \infty$, and these are reflections of the infinite-order phase transition at $\Delta=1$ in the 'true' system. Note that the upper branch of the SUB2-m results are physical and the lower branch is unphysical

of the lowest-lying excited state for the spin-half $X X Z$ model on the square lattice.

We find that for all LSUBm approximations with $m>2$ the physical branch of ground-state solutions (i.e. the one which becomes exact in the $\Delta \rightarrow \infty$ limit) terminates at a critical value $\Delta_{r}$, such that for $\Delta<\Delta_{\text {c }}$ wo real solution exists. These LSUBm "critical points" are analogous to the SUB2-m limit points of the previous subsection, and they are again taken to be a signal of the phase transition at (or near to) $\Delta=1$. (Note that the "unphysical" LSUBm branches, as seen for the SUB2-m approximations above, are not plotted here in order to present a clear illustration of our results. although they rertainly exist.)

The SUB2 and ISUBm results using the z-aligned state as model state are compared in Table 7.1 for the isotropic $(\Delta=1)$ case with results from linear spin-wave theory (LSWT) [74], series expansion techniques [75], and quantum Monte Carlo (QMC) simulations [76]. Figures 7.2 and 7.4 show the corresponding results for the ground-state energy and lowest-lying excitation energy $\epsilon_{0}$ as functions of $\Delta$. Our results for the ground-and excited-state properties of the $X X Z$ systems are seen to be in excellent agreement with those results of the best of other approaches. We also note that values for the spin stifhess of the Heisenberg model (see [77] and later on in this Chapter for 
further details) are also found to be very accurate. Furthermore, calculations have been carried out for this model using an extended version of the CCM in which the bra-state correlation operator $\tilde{S}$ is also written in an exponentiated form, analogous to that of the ket-state operator. The interested reader is referred to $[61]$ for more details.

\subsubsection{CCM Results for the Spin-Half Square-Lattice $X X Z$ Model Using a Planar Model State}

There is never a unique choice of model state $|\Phi\rangle$. Indeed, our choice should be guided by any physical insight available to us concerning the system or, more specifically, that particular phase of it which is under consideration. In the absence of any other insight into the quantum many-body system, we may sometimes be guided by the behaviour of the corresponding classical system. The $X X Z$ model under consideration provides just such an illustrative example. Thus, for $\Delta>1$ the classical Hamiltonian of (7.13) on the 2D square lattice (and, indeed, on any bipartite lattice) is minimized by a perfectly antiferromagnetically Néel-ordered state in the $z$-direction, and we have already utilised this information in the preceding subsections. However, the classical ground-state energy is minimized by a Nél-ordered state with spins pointing

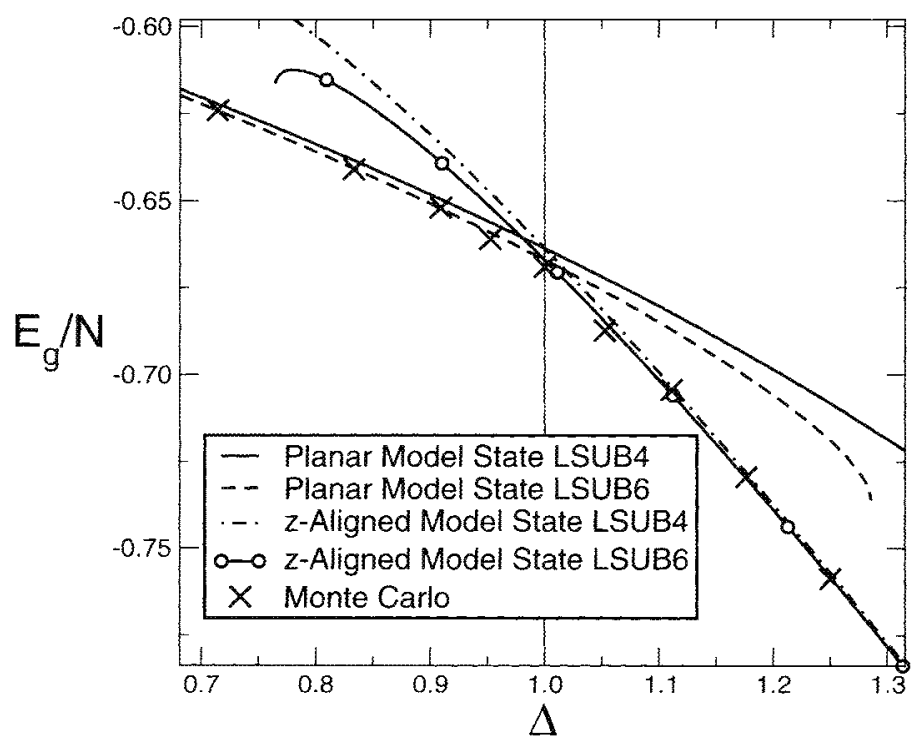

Fig. 7.2. CCM LSUBm results using the z-aligned and planar Néel model states for the ground-state energy of the spin-half square-lattice $X X Z$ model compared to quantum Monte Carlo results of [73]. Results for the LSUB6 approximation using both model states end at their respective critical points 


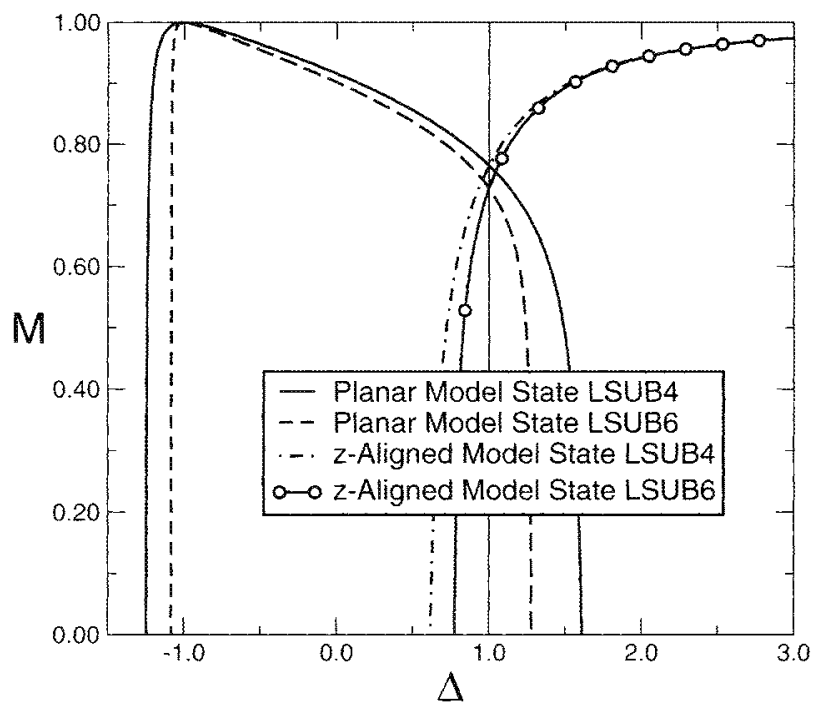

Fig. 7.3. CCM LSUBm results using the z-aligned and planar Neel model states for the sublattice magnetisation of the spin-half square-lattice $X X Z$ model. Results for the LSUB4 and LSUB6 approximation using both model states end at their respective critical points

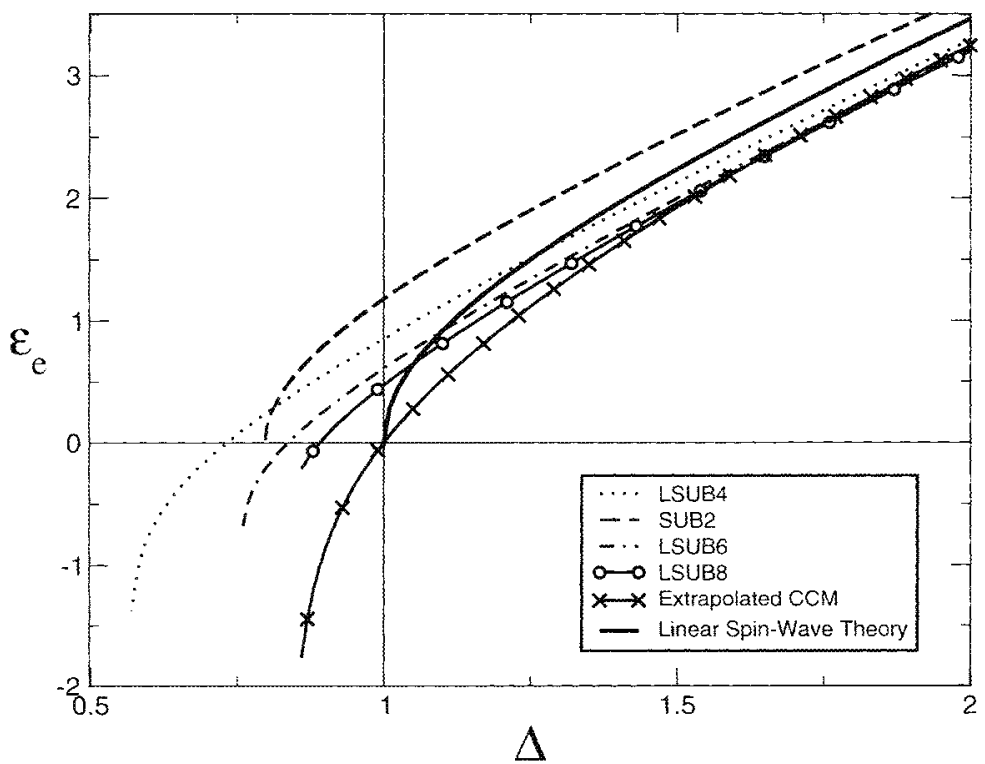

Fig. 7.4. CCM LSUBm results using the z-aligned Néel state as model state for the lowest-lying excitation energies of the spin-half square-lattice $X X Z$ model 
Table 7.1. CCM results $[59,62]$ for the isotropic $(\Delta=1)$ spin-half square-lattice Heisenberg antiferromagnet compared to results of other methods. The numbers of fundamental configurations in the ground-state and excited-state CCM wave functions for the $z$-aligned Néel model state are given by $N_{f}^{z}$ and $N_{f e}^{z}$, respectively, and the number of fundamental configurations in the ground-state CCM wave function for the planar Néel model state is given by $N_{f}^{p}$. Results for the critical points of the $z$-aligned Néel model state are indicated by $\Delta_{c}^{z}$ and results for the critical points of the planar Néel model state are indicated by $\Delta_{c}^{p}$. (Note that results for the ground-state expectation values for both model states are identical for the isotropic Heisenberg model at $\Delta=1$ )

\begin{tabular}{|l|c|c|c|c|c|c|c|c|}
\hline Method & $E_{g} / N$ & $M$ & $\epsilon_{e}$ & $N_{f}^{z}$ & $N_{f_{c}}^{z}$ & $N_{f}^{p}$ & $\Delta_{c}^{z}$ & $\Delta_{c}^{p}$ \\
\hline \hline LSUB2 & -0.64833 & 0.841 & 1.407 & 1 & 1 & 1 & & - \\
\hline SUB2 & -0.65083 & 0.827 & 1.178 & - & & & 0.799 & 1.204 \\
\hline LSUB4 & -0.66366 & 0.765 & 0.852 & 7 & 6 & 10 & 0.577 & 1.648 \\
\hline LSUB6 & -0.66700 & 0.727 & 0.610 & 75 & 91 & 131 & 0.763 & 1.286 \\
\hline LSUB8 & -0.66817 & 0.705 & 0.473 & 1273 & 2011 & 2793 & 0.843 & - \\
\hline Extrapolated CCM & -0.6697 & 0.62 & 0.00 & & & & 1.03 & \\
\hline LSWT [74] & -0.658 & 0.606 & 0.0 & - & - & $\cdots$ & 1.0 & $\cdots$ \\
\hline Series Expansions [75] & $-0.6693(1)$ & $0.614(2)$ & - & - & $\cdots$ & $\cdots$ & - & $\cdots$ \\
\hline QMC [76] & $-0.669437(5)$ & $0.6140(6)$ & - & - & $\cdots$ & & $\cdots$ & $\cdots$ \\
\hline
\end{tabular}

along any direction in the $x y$ plane, say along the $x$-axis for $-1<\Delta<1$. Thus, in order to provide CCM results in the region $-1<\Delta<1$, we now take this state to be our model state and we shall refer to it as the "planar" model state.

In order to produce another "ferromagnetic" model state for the planar model state in the local frames, we rotate the axes of the left-pointing spins (i.e., those pointing in the negative $x$-direction) in the planar state by $90^{\circ}$ about the $y$-axis, and the axes of the corresponding right-pointing spins by $-90^{\circ}$ about the $y$-axis. (Note that the positive $z$-axis is defined here to point upwards and the positive $x$-axis is defned to point rightwards.) Thus, the transformations of the local axes are described by

$$
s^{x} \rightarrow s^{z}, \quad s^{y} \rightarrow s^{y}, \quad s^{z} \rightarrow-s^{x}
$$

for the left-pointing spins, and by

$$
s^{x} \rightarrow-s^{z}, \quad s^{y} \rightarrow s^{y}, \quad s^{z} \rightarrow s^{x}
$$

for the right-pointing spins. The transformed Hamiltonian of (7.13) may now be written in these local axes as

$$
H=-\frac{1}{4} \sum_{\langle i, j\rangle}\left[(\Delta+1)\left(s_{i}^{+} s_{j}^{+}+s_{i}^{-} s_{j}^{-}\right)+(\Delta-1)\left(s_{i}^{+} s_{j}^{-}+s_{i}^{-} s_{j}^{+}\right)+4 s_{i}^{z} s_{j}^{z}\right],
$$


In this case we track the CCMI solution for the planar model state from the point $\Delta=-1$. We note that all of the $\mathrm{CCN}$ correlation coefficients are zero at $\Delta=-1$ because the model state is an exact ground eigenstate of the Hamiltonian of (7.33) at this point. The results for the grome-state energy using the planax model state are plotted in Fig. 7.2. and the corresponding results for the sublattice magnetisation ( $M$. again defined with respect to the rotated local spin axes) are shown in Fig. 7.3. Furthermore. we note that the Hamiltonian for the planar model state of (7.33) is identical to the Hamiltonian for the a-aligned model state of (7.17) at $\Delta=1$. Indeed, we obtain identical results for the gromelstate expextation values at $\Delta=1$. and this is an excellent test of the validity of our results.

\subsubsection{Quantum Criticality of the Antiferromagnetic Phase Transition for the Spin-Half Square-Lattice $X X Z$ Model}

We wish to investigate the quantum criticality of the phase at (or near to) $\Delta=1$ for the case of the square latice. The critical index for the singular (non-analytic) term in $E_{g} / N$ near an LSUBm critical point $\Delta,(m)$ can first be obtained, for example. by direct examination of the anisotropy susceptibility. $\gamma_{n} \equiv-\partial^{2}\left(E_{\eta,} / N\right) / \partial \Delta^{2}$. For $m>2$ we find.

$$
\chi_{a}^{m}(\Delta) \rightarrow \chi_{a}^{m}\left|\Delta-\Delta_{r}(m)\right|^{-x_{(3)}}: \Delta \rightarrow \Delta_{c}(m) .
$$

Direct calculation for the ISUBm approximations using both the $z$-aligned and planar Neel model states shows that for $m>2$ we have $\alpha_{0} \approx 1.500 \pm$ 0.005 . However, the prefactors $\chi_{r i}^{\prime \prime}$ in $(7.34)$ are themselves strongly dependent on the truncation index $m$. We may now use a variant of the so-called coherent anomaly method (CAMI) of Swzuki [78] to extract further information. Thus, we attempt to fit $\bar{X}_{a}^{m}$ with the coherent anomaly form,

$$
x_{a}^{m} \rightarrow K\left|\Delta_{c}(\infty)-\Delta_{c}(m)\right|^{\nu^{\prime}}: \Delta \rightarrow \Delta_{c}(\infty)
$$

where $f$ is a constant. Thus, as explained by Suzuki [78]. one may intuit or prove that the exact $\chi_{a}(\Delta)$ has the critical form,

$$
\chi_{u}(\Delta) \rightarrow r\left|\Delta-\Delta_{c}(\infty)\right|^{-n_{0}+\nu}: \quad \Delta \rightarrow \Delta_{f}(\infty) \equiv \Delta_{c} .
$$

where $k$ is a constant.

A CAM analysis along these lines of the LSUBm results based on the $z$-aligned Néel state gives $\nu \approx 1.25$ using the $\Delta_{A}^{z}(4)$ and $\Delta_{A}^{z}(6)$ data, and $\nu \approx 0.97$ using the $\Delta_{A}^{z}(6)$ and $\Delta_{A}^{z}(8)$ data. We thus obtain a singular term in $E_{g} / N$ near $\Delta_{A}^{z}$ with a critical exponent $2-\alpha_{0}+\nu \approx 1.50-1.75$. This may be compared with the corresponding value of $3 / 2$ for both the mean-fieldlike CCM SUB2 approximation (in which all 2-spin-flip correlation terms are retained, however far apart on the lattice) and linear spin-wave theory (LSWT). A similar treatment for the planar model state yields a critical exponent of $2-\alpha_{0}+\nu \approx 1.77$. which is in good agreoment with the result for the z-aligned Néel model state. 


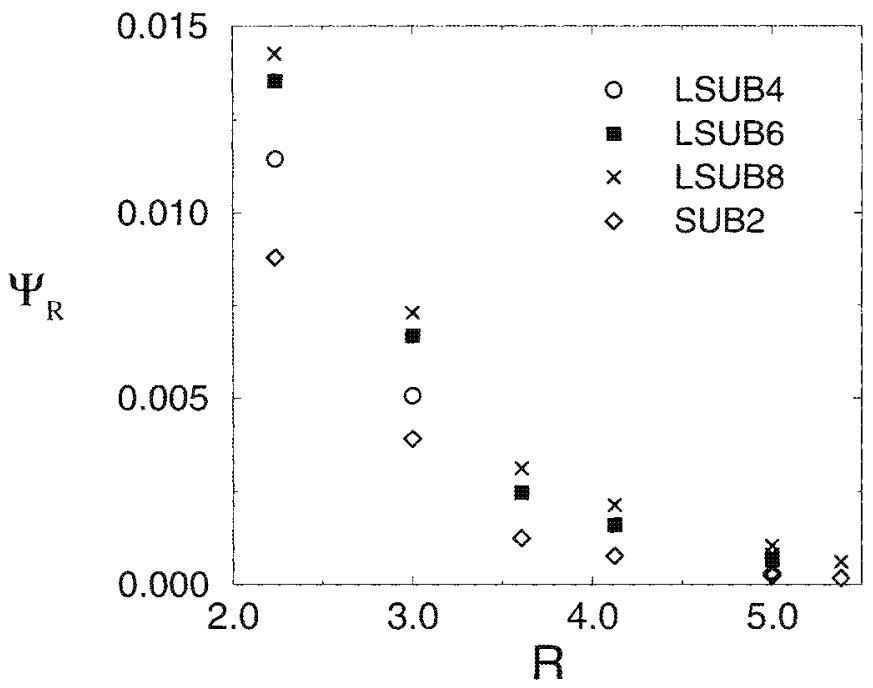

Fig. 7.5. Results for the Ising-expansion coefficients, plotted as a function of the: lattice distance $R$, corresponding to two-body excitations with respect to the model state for the spin-half, square lattice Heisenberg model at $\Delta=1$ obtained via the LSUB $m$ approximation scheme (with $m=\{4,6,8\}$ ) and the SUB2 approximation. (Figure taken from [32])

\subsubsection{CCM Prediction of the Nodal Surface of the Spin-Half Square-Lattice Heisenberg Model}

We consider an expansion of the ground-state wave function in a complete Ising basis $\{|I\rangle\}$ (in terms of the local coordinates after rotation). This may be again written as, $|\Psi\rangle=\sum_{I} \Psi_{I}|I\rangle$, where the sums over $I$ goes over all $2^{N}$ Ising states, and we find that this expression naturally leads from (7.2) (also see $[32,60])$ to an exact mapping of the CCM correlation coefficients $\left\{\mathcal{S}_{I}\right\}$ to the Ising-expansion coefficients $\left\{\Psi_{l}\right\}$, which is given by

$$
\Psi_{I}=\left\langle\Phi\left|C_{I}^{-} e^{s}\right| \Phi\right\rangle \equiv\left\langle\Phi\left|s_{i_{1}}^{-} s_{i_{2}}^{-} \cdots s_{i_{1}}^{-} e^{s}\right| \Phi\right\rangle
$$

It is possible to match the terms in the exponential to the 'target' configuration of $C_{I}^{-}$in (7.37), and so obtain a mumerical value for the $\left\{\Psi_{I}\right\}$ coefficients once the CCM ket-state equations have been derived and solved for a given value of the anisotropy. Note that we may plot the Ising-expansion coefficients as a function of the lattice distance $R$, corresponding to two-body excitations with respect to the model state, and results are shown in Fig. 7.5.

We observe that all of the coefficients are found to be positive. and this shows that the exact Marshall-Peierls sign rule is being obeyed for our $a b$ initio calculation. We note that no such condition is imposed in our CCM treatment of this model. Indeed, it is also the case that all other four- or 
higher-body terms have corresponding Ising expansion terms which are positive. We also note that the Ising expansion coefficients appear to converge rapidly with increasing levels of approximation, and that a strength of the CCM is that it may be applied to even very strongly frustrated systems where no analognes of the Marshall-Peierls sign rule are usually known.

We note that it might be possible to use the CCM in order to simulate aceurately the nodal surface of quantum problem and this information might be fod into a fixed-node QNC calculation in order to simulate very accurately the properties of this system. Indeed. general rules might be inferred from the CCM data and, if so. an exact solution. to within QMC statistical limits. might be determined. The interested reader is referred to $[31.32 .60]$ for more information.

\subsection{The $\boldsymbol{J}-\boldsymbol{J}^{\prime}$ Model: A Square-Lattice Model with Competing Nearest-Neighbour Bonds}

We now wish to show that the CCM can treat frustrated systems as easily as unfrustrated systems, and we begin by noting that the $J J^{\prime}$ model is a spinhalf Heisenberg nodel on a square lattice with two kinds of nearest-neighbour bonds $J$ and $J^{\prime}$. as shown in Fig. 7.6 .

$$
H=J \sum_{\langle i\rangle_{1}} \mathbf{s}_{i} \cdot \mathbf{s}_{j}+J^{\prime} \sum_{\langle i j\rangle_{2}} \mathbf{s}_{i} \cdot \mathbf{s}_{j} \cdot
$$

The sums over $\langle i j\rangle_{1}$. and $\langle i j\rangle_{2}$ represent sums over the nearest-neighbour bonds show in Fig. 7.6 by dashed and solid lines respectively. Each square-

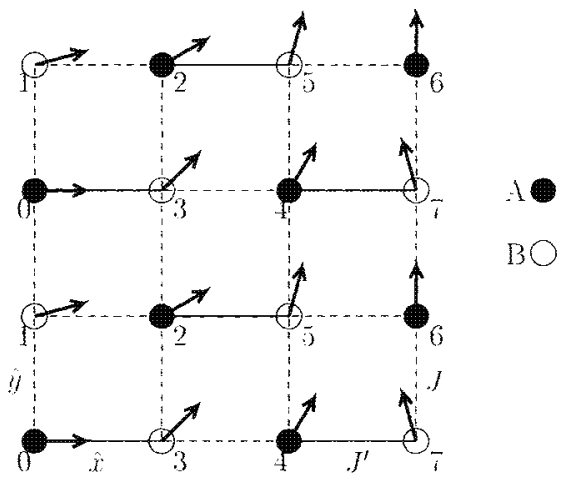

Fig. 7.6. Illustration of the $J J^{\prime}$ model of (7.38), with two kinds of regularly distributed nearest-neighbour exchange bonds. $J$ (dashed lines) and $f^{\prime}$ (solid lines) and its classical spiral state $(\Phi>0)$ shown for the ferromagnetic case $(J<0$. $\left.J^{\prime}>|J| / 3\right)$. (Figure taken from $[77]$ ) 
lattice plaquette consists of three $J$ bonds and one $J^{\prime}$ bond. A model with such a zigzag pattern has been treated by various methods $[64,77,79-82]$. For the cases in which $J$ and $J^{\prime}$ have different signs (i.e., one bond is ferromagnetic while the other is antiferromagnetic) the plaquettes are frustrated, whereas competition without frustration is realized for antiferromagnetic bonds $\left(J^{\prime}\right\rangle$ 0 and $f>0$ ).

Using this model we discuss the influence of quantum fluctuations on the ground-state phase diagram and in particular on the nature of the zerotemperature phase transitions from phases with collinear magnetic order at small frustration to phases with noncollinear spiral order at large frustration. The role of quantum fuctuations is examined by comparing ferromagneticspiral and antiferromagnetic-spiral transitions within the same nodel. Whereas for the classical version of the $J \cdot J^{\prime}$ model both situations can be mapped onto each other, the quantum model behaves differently in the two cases and this is because of the different nature of the collinear state. The quantum Néel state on two-dimensional lattices exhibits strong quantum fluctuations. For example, as we saw in the previous section, the sublattice magnetisation of the Heisenberg antiferromagnet (HAF) on the square lattice is only about $60 \%$ of its classical value. By contrast, the ferromagnetic state is the same for the quantum and the classical model and there are no quantum fluctuations in this state.

The classical ground state of this $J-J^{\prime}$ model is collinear (i.e., ferromagnetic or antiferromagnetic depending on the sign of $J$ ) for the unfrustrated cases. For $\left|J^{\prime}\right|>|J| / 3$ (and $J$ and $J^{\prime}$ having different signs) the frustration is large enough in order to force the ground state to be a noncollinear state of spiral nature with a characteristic pitch angle $\Phi= \pm\left|\Phi_{\text {c1 }}\right|$ given by

$$
\left|\Phi_{c l}\right|=\left\{\begin{array}{ll}
0 & \left|J^{\prime}\right|<\frac{|J|}{3} \\
\arccos \left(\frac{1}{2} \sqrt{1+\frac{1}{\left|J^{\prime}\right|}}\right) & \left|J^{\prime}\right| \geq \frac{|J|}{3}
\end{array} .\right.
$$

Figure 7.6 shows the classical spiral state for the ferromagnetic case $(J<$ $\left.0, f^{\prime}>|J| / 3\right)$ where the spin orientations at $\mathrm{A}$ and $\mathrm{B}$ lat tice sites as numbered on the figure are defined the angle $\theta_{n}=n \Phi_{a\}}$. For the antiferromagnetic case $\left(J>0, J^{\prime}<-J / 3\right)$ all of the spins on one sublattice are reversed. We note that $\Phi=0$ corresponds to the collinear state. The classical transition between the collinear and the noncollinear state is of second order and takes place at the critical point $J_{c}^{\prime}=-J / 3$. Figure 7.7 gives an illustration of the complete classical ground-state phase diagram.

We choose the spiral state with the characteristic angle $\Phi$ (illustrated in Fig. 7.6) as our CCM model state. Further details concerning the treatment of the $J-J^{\prime}$ model via the CCM are given in $[64,77,81]$. Wo calculate the ground state and the low-lying excitations of the Hamiltonian of (7.38). We use the CCM for high orders of approximation up to LSUB8 which contains 4986 fundamental configurations for the Néel model state with $\Phi=0$ and 42160 fundamental configurations for a helical model state with $\phi \neq 0$. (Wo 


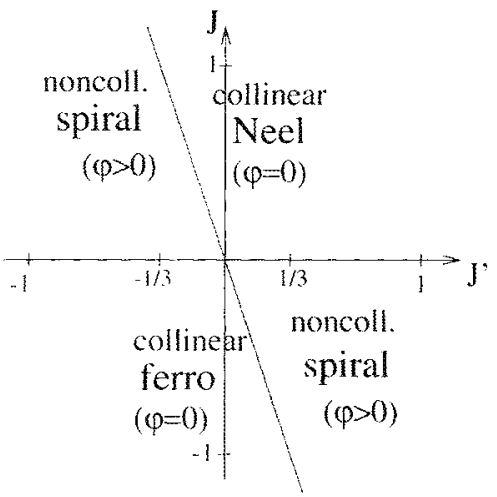

Fig. 7.7. Classical grounci-state phase diagram for the $f f^{\prime}$ model on the square lattice with competing nearest-neighbour bonds, indicating the collinear Néel and ferromagnetic phases and the noncollinear spiral phases for various values of $J$ and $J^{\prime}$. (Figure taken from [77])

note that such a large number of configurations as the latter case may be considered only by using parallel processing techniques, althongh this is not performed here. The interested reader is referred to [83] for more details of a parallelised implementation of the CCN.) By way of comparison we also exactly diagonalise finite sized lattices of up to $N=32$ spins with periodic boundary conditions. We extrapolate to the infinite-lattice limit using standard finite-size scaling laws.

For sufficiently strong antiferromagnetic $J^{\prime}$ bonds the $J . J^{\prime}$ model is characterised by a tendency to singlet pairing of the two spins coupled by a $f^{\prime}$ bond, and hence the long-range magnetic (collinear or noncollinear) order is destroved. We observe clear indications of a second-order phase transition to a guantmon paramagnetic dimerised phase at a certain crit ical value of $J^{\prime}=J^{\prime}$. However the only case exammed in detail here is the antiferromagnetic case $(J=+1)$. Evidence of a phase transition to a dimerised phase is indicated by the sublattice magnetisation (see Fig. 7.8). The results of the CCN and exact diagonalisations (ED) agree well with each other and with the result $J_{s}^{\prime} \approx 2.56$ from cumulant series expansions [79], whereas by contrast renormalised spin wave theories (RSWT) clearly overestimate the order. We also note that another indication of a dimerised phase is the appearance of a gap $\Delta$ between the singlet ground state and the first triplet excitation. The gap appears to open in the range $2.5 \leqslant J_{s}^{\prime} \leq 3.0$ for both the ED and CCN calculations (see Fig. 7.8). This result is in good agreement with the corresponding estimates for the critical point using the magnetisation.

The phase transition to the dimerised phase is also indicated by the spin stiffness $\rho_{s,}$. The ground-state stiffness is a variable which indicates the distance of the ground state from criticality and the breakdown of the Need long-range order is thus accompanied by $\rho_{s}$ going to zero. The spin stiffness 

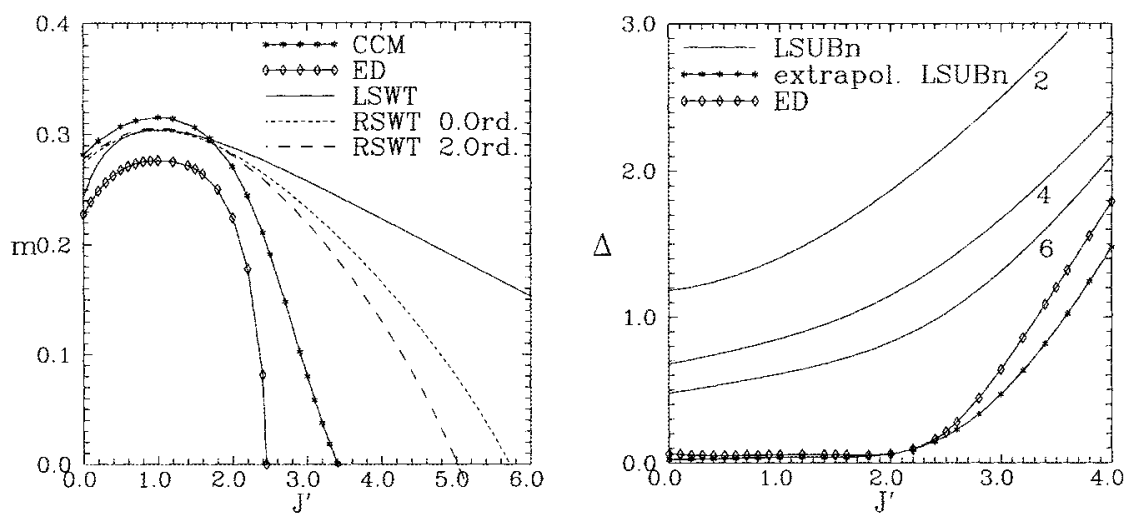

Fig. 7.8. Indications of a phase transition to the dimerised phase for the $J-J^{\prime}$ model on the square lattice with competing nearest-neighbour bonds, with $J=+1$. Left graph: sublattice magnetisation versus $J^{\prime}$ using the CCM, exact diagonalisation, and spin wave theories. Right graph: spin gap versus $J^{\prime}$ using the CCM compared to results of exact diagonalisation. (Figure taken from [77])

measures the amount of energy used in introducing a twist $\theta$ to the direction of spin between every pair of neighbouring rows, such that

$$
\rho_{s}=\left.\frac{d^{2}}{d \theta^{2}} \frac{E_{0}(\theta)}{N}\right|_{\theta=0},
$$

and this quantity may be calculated directly using the CCM.

We note that the magnetic order parameters may only tell us whether certain types of long-range order are present, whereas the spin stiffness has the advantage of being unbiased with respect to the nature of the ordering. The spin stiffness constitutes, together with the spin wave velocity, the fundamental parameter that determines the low-energy dynamics of magnetic systems [84]. The CCM LSUBn results are given in Fig. 7.9. We calculate the stiffness using two different directions of in-plane rows, i.e., rows parallel to the $J^{\prime}$ bonds and rows perpendicular to the $J^{\prime}$ bonds. We note that, although the results of the stiffness for the two directions are different in general (see Fig. 7.9), the phase transition points (i.e., the values of $J^{\prime}$ where $\rho_{s}$ becomes zero) agree well with each other for the various LSUBn approximations although the extrapolated CCM results are expected to be even more accurate. Our calculations predict that $J_{s}^{\prime} \approx 2.8$ which is again in good agreement with the results of the other methods. We note that this phase transition to the dimerised phase is expected to belong to the three-dimensional $O(3)$ universality class as indicated by the value of the correlation length critical exponent [82].

We now consider the frustrated region of the $J-J^{\prime}$ model for $J$ and $J^{\prime}$ with different signs. We note that classically there is a second-order phase transition from collinear order to noncollinear order at $J^{\prime}=-J / 3$ for both 

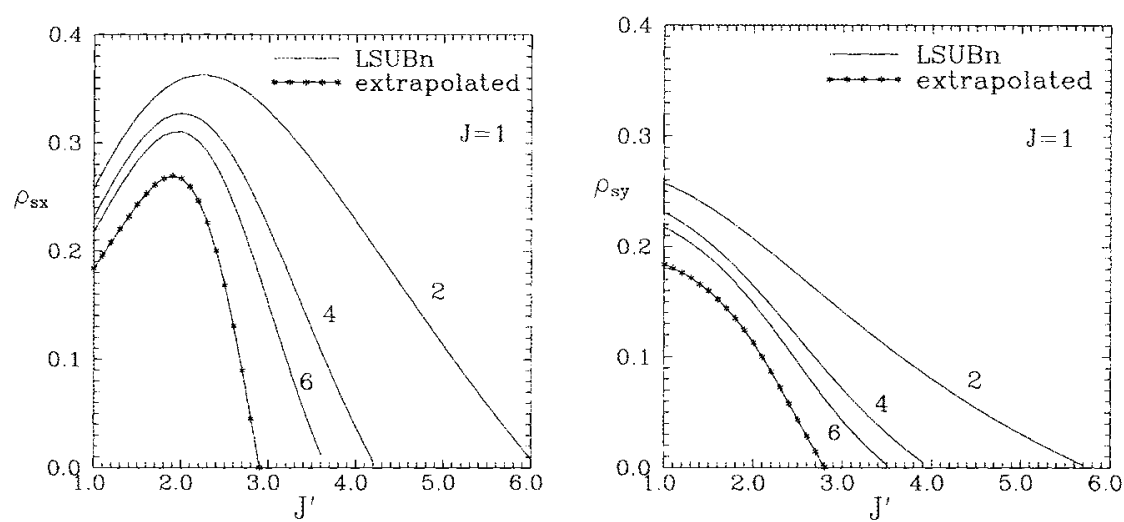

Fig. 7.9. The spin stiffness versus $I^{\prime}$ for two different rows within the plane of the lattice are good indicators of a phase transition to the dimerised phase for the $J$... $J^{\prime}$ model on the square lattice with competing nearest-neighbour bonds, with $J=+1$. Left graph: rows along the $x$ direction (parallel to the $J^{\prime}$ bonds). Right graph: rows along the $y$ direction (perpendicular to the $f^{\prime}$ bonds). (Figure taken from [77])

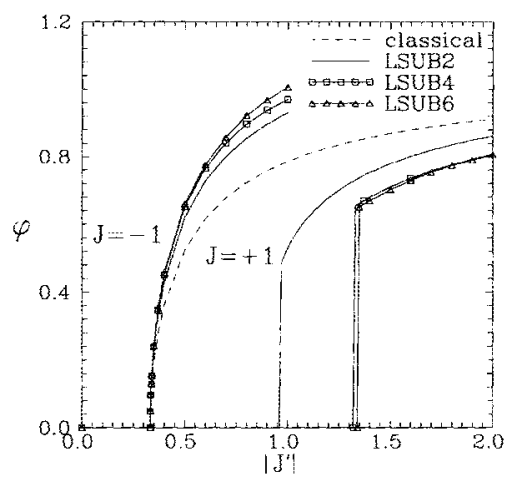

Fig. 7.10. Pitch angle $\Phi$ versus $\left|J^{\prime}\right|$ for the quant um and the classical case of the $J$ $J^{\prime}$ model on the square lattice with competing nearest-neighbour bonds. Although $\Phi$ is classically the same for the ferromagnetic case $\left(J=-1, J^{\prime}>0\right)$ and for the antiferromagnetic case $\left(J=+1, J^{\prime}<0\right)$ we note that the quantum pitch angle is different for both cases. The curves to the left of the classical (dashed) curve belong to $J=-1$ and those to the right of it belong to $J=+1$. (Figure taken from [77])

antiferromagnetic and ferromagnetic nearest-neighbour $J$-bonds. By contrast, the behaviour of the quantum model for the two cases is different concerning the phase transition. In particular. we find that the critical point is shifted to $J^{\prime} \approx-1.35$ (see Fig. 7.10) for the antiferromagnetic case $(J=+1)$, although no such shift is observed for the ferromagnetic case $(J=-1)$.

The exact diagonalisation (ED) data of the structure factor $S(\mathbf{k}$ ) (see Fig. 7.11) also agree with these findings. For $J=+1$ the collinear Néel order 

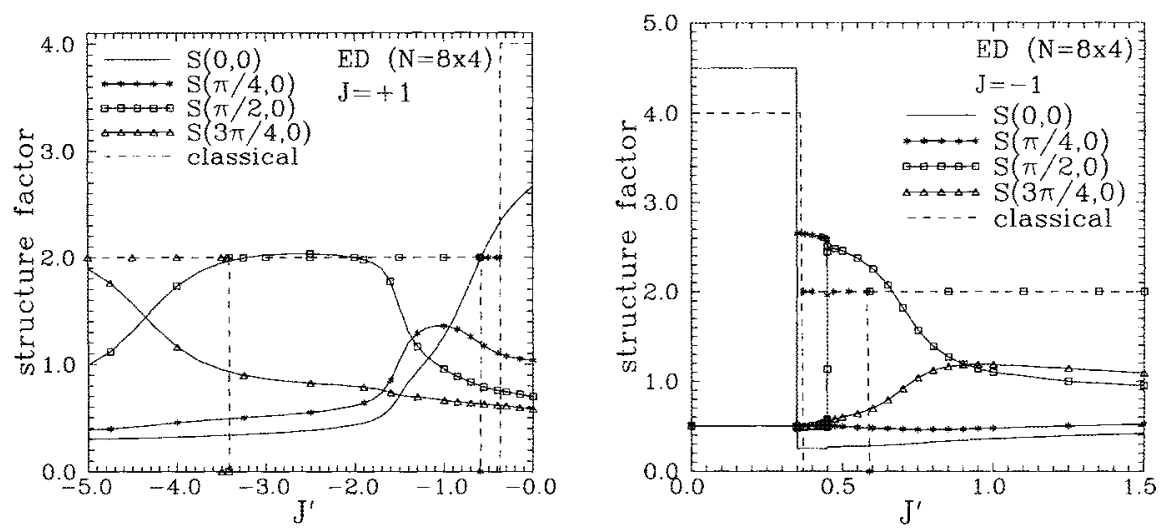

Fig. 7.11. Ground-state structure factor $S(\mathbf{k}) \propto \sum_{i, j \in A} e^{i\left(\mathbf{R}_{j}-\mathbf{R}_{i}\right) \cdot \mathbf{k}}\left\langle\mathbf{s}_{i} \cdot \mathbf{s}_{j}\right\rangle$ (note that the indices $i$ and $j$ run over one sublattice) for a $8 \times 4$ lattice of the $J-J^{\prime}$ model on the square lattice with competing nearest-neighbour bonds for the quantum and the classical case for various spiral vectors $k$ for antiferromagnetic $J=+1$ (left graph) and ferromagnetic $J=-1$ (right graph). (Figure taken from [77])

$[\mathbf{k}=(0,0)]$ becomes unstable in comparison to the noncollinear spiral order $[\mathbf{k}=(\pi / 4,0)]$ in the classical model for $J^{\prime} \lesssim-0.36$. We note that this occurs only for $J^{\prime} \lesssim-0.95$ in the quantum case. The situation for the ferromagnetic case $(J=-1)$ is again different, and the results of the structure factor show that the transition from $k=(0,0)$ (collinear ferromagnetic order) to $\mathrm{k}=(\pi / 4,0)$ (spiral order) takes place at nearly the same value of $J^{\prime} \approx 0.36$ for both the classical and the quantum cases. We may also use the difference between the amount of the on-site magnetic moment $\left\langle s_{i}\right\rangle$ and its classical value $\left\langle s_{i}\right\rangle_{\mathrm{d}}=1 / 2$ as a measure of quantum fluctuations. We compare the strength of quantum fluctuations near the collinear-noncollinear transitions for both the antiferromagnetic and the ferromagnetic cases. Although the quantum fuctuations are particularly strong for $I=+1$ near the antiferromagneticspiral transition (leading to an on-site magnetic unoment less then $20 \%$ of its classical value [64]), there are virtually no quantum fluctuations at the ferromagnetic-spiral transition for $J=-1$ because the on-site magnetic moment takes its classical value $1 / 2$ up to $J^{\prime} \approx 0.36$ ( $\mathrm{cf}$. [81]). Hence the shift of the critical $J_{c:}^{\prime}$ in the antiferromagnetic case can clearly be attributed to the strong quantum fluctuations.

Wo may summarise by saying that our findings are generally consistent with the statement that quantum fuctuations (which we have in the antiferromagnetic case only) prefer a collinear ordering. We note that the quantum collinear state can survive for the quantum model studied here into a classically frustrated region in which classical theory indicates that the collinear state is already unstable. In addition, our results indicate that there is a second-order phase transition for the ferromagnetic case $(J=-1)$ which is 

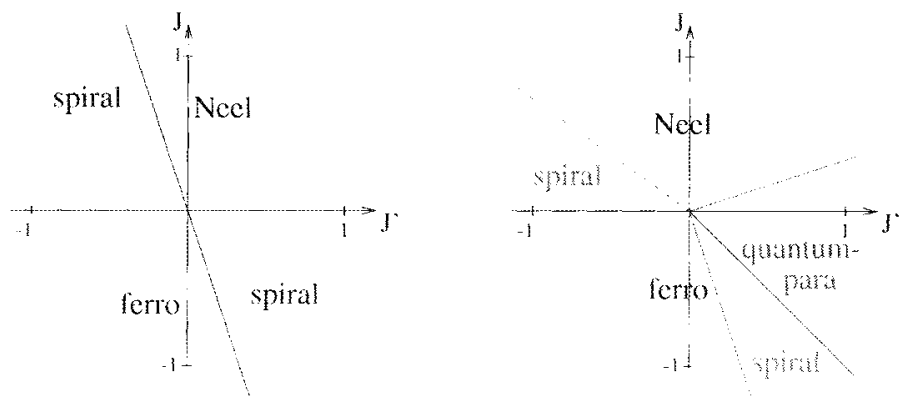

Fig. 7.12. Phase diagrams of the $J J^{\prime}$ model on the square lattice with competing nearest-neighbour bonds for the classical case (left graph) and for the quantum (ase (right graph). The dashed line indicates a first-order phase transition, while the other transitions are of second order. (Figure taken from [77])

in agrement with classical theory. The collinear-noncollinear transition in the antiferromagnetic case $(J=+1)$ is probably of first order for the quant um model (cf. Fig. 7.10 and discussion in [64]) which compares to a second-order transition for the classical case. Figure 7.12 compares the phase diagrams of this $J \cdot J^{\prime}$ model for the classical and the quantum cases.

\subsection{An Interpolating Kagomé/Triangle Model}

We also wish to study another strongly frustrated spin-half Heisenberg model. namely one which interpolates smoothy between the triangular-lattice antiforomagnet (TAF) [59.85 87] and the Kagomé-lattice [65.88.89] antiferromagnet (KAF). We slall refer to this as the interpolating Kagomé/triangle model (illustrated in Fig. 7.13), and the Hamiltonian is given by

$$
H=J \sum_{\langle i, j\rangle} \mathrm{s}_{i} \cdot \mathrm{s}_{j}+. J^{\prime} \sum_{\{i, k\}} \mathrm{s}_{i} \cdot \mathrm{s}_{k} .
$$

where $\langle i . j\rangle$ rums over all nearest-neighbour (n.n.) bonds on the Kagomé lattice and $\{i . k\}$ rums over all n.n. bonds which comnect the Kagome lattice sites to those other sites on an underlying triangular lattice. Note that each bond is comted once and once only. We explicitly set $J=1$ throughout this paper. and we note that at $J^{\prime}=1$ we thus have the $\mathrm{TAF}$ and at $J^{\prime}=0$ we have the KAF.

\subsubsection{CCM Treatment of the Interpolating Kagomé/Triangle Model}

For the interpolating Kagomé/triangle model described by (7.41). we choose a model state $|\Phi\rangle$ in which the lat tice is divided into three sublattices. denoted $\{$ A.B.C $\}$. The spins on sublattice $A$ are oriented along the nogative 


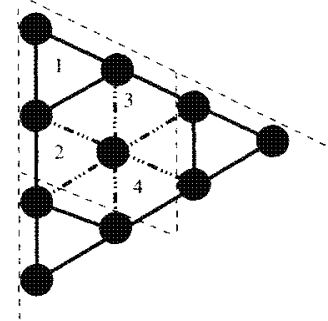

(a)

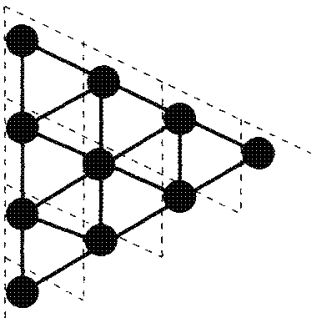

(b)

Fig. 7.13. The interpolating Kagomé/triangle model is illustrated in diagram (a), where the bonds of strength $J$ between Kagome lattice sites are indicated by the thick solid lines and the non-Kagomé bonds of strength $J^{\prime}$ on the underlying triangular lattice sites are indicated by the "broken" lines. The triangular lattice Heisenberg antiferromagnet (TAF) is illustrated in diagram (b), and we note that the two models are equivalent when $J=J^{\prime}$. The quadrilateral unit cells for both cases are also illustrated. The interpolating Kagomé/triangle model contains four sites per unit cell, whereas the TAF has only one site per unit cell. (Figure taken from [65])

$z$-axis, and spins on sublattices $B$ and $C$ are oriented at $+120^{\circ}$ and $-120^{\circ}$. respectively, with respect to the spins on sublattice $A$. Our local axes are chosen by rotating about the $y$-axis the spin axes on sublattices $B$ and $C$ by $-120^{\circ}$ and $+120^{\circ}$ respectively, and by leaving the spin axes on sublattice $A$ unchanged. Under these canonical transformations.

$$
\begin{aligned}
s_{B}^{x} \rightarrow-\frac{1}{2} s_{B}^{x}-\frac{\sqrt{3}}{2} s_{B}^{z} ; s_{C}^{x} & \rightarrow-\frac{1}{2} s_{C}^{x}+\frac{\sqrt{3}}{2} s_{C}^{z}, \\
s_{B}^{y} \rightarrow s_{B}^{y} ; s_{C}^{y} & \rightarrow s_{C}^{y}, \\
s_{B}^{z} \rightarrow \frac{\sqrt{3}}{2} s_{B}^{x}-\frac{1}{2} s_{B}^{z} ; s_{C}^{z} & \rightarrow-\frac{\sqrt{3}}{2} s_{C}^{x}-\frac{1}{2} s_{C}^{z} .
\end{aligned}
$$

The model state $|\Phi\rangle$ now appears mathematically to consist purely of spins pointing downwards along the $z$-axis, and the Hamiltonian (for $J=1$ ) is given in terms of these rotated local spin axes as;

$$
\begin{aligned}
H & =\sum_{\langle i \rightarrow j\rangle}\left\{-\frac{1}{2} s_{i}^{z} s_{j}^{z}+\frac{\sqrt{3} \lambda}{4}\left(s_{i}^{z} s_{j}^{+}+s_{i}^{z} s_{j}^{-}-s_{i}^{+} s_{j}^{z}-s_{i}^{-} s_{j}^{z}\right)\right. \\
& \left.+\frac{\lambda}{8}\left(s_{i}^{+} s_{j}^{-}+s_{i}^{-} s_{j}^{+}\right)-\frac{3 \lambda}{8}\left(s_{i}^{+} s_{j}^{+}+s_{i}^{-} s_{j}^{-}\right)\right\} \\
+y^{\prime} & \sum_{\{i \rightarrow k+}\left\{-\frac{1}{2} s_{i}^{z} s_{i}^{z}+\frac{\sqrt{3} \lambda}{4}\left(s_{i}^{z} s_{k}^{+}+s_{i}^{z} s_{k}^{-}-s_{i}^{+} s_{k}^{z}-s_{i}^{-} s_{k}^{z}\right)\right. \\
& \left.+\frac{\lambda}{8}\left(s_{i}^{+} s_{k}^{-}+s_{i}^{-} s_{k}^{+}\right)-\frac{3 \lambda}{8}\left(s_{i}^{+} s_{k}^{+}+s_{i}^{-} s_{k}^{-}\right)\right\} .
\end{aligned}
$$


Note that $i$ and $j$ rum only over the $N_{k}$ sites on the Kagome lattice. whereas $k$ runs over those non-Kagomé sites on the (underlying) triangular lattice. $N$ indicates the total number of triangular-lattice sites, and each bond is counted once and once only. We also note that we have multiplied all of the off-diagonal terms in the new Hamiltonian by a factor of $\lambda$. We shall use this factor in order to determine the perturbation series around the Ising limit $(\lambda=0)$ for the gromd-state energy and sublattice magnetisation. The case $\lambda=1$ corresponds to our isotropic Heisonberg case of (7.41). The symbol $\rightarrow$ indicates an explicit bond directionality in the Hamiltonian given by (7.43). namely, the three directed nearest-neighbou bonds included in (7.43) point from sublattice sites $\mathrm{A}$ to $\mathrm{B}$. B to $\mathrm{C}$. and $\mathrm{C}$ to $\mathrm{A}$ for both types of bond. We now perform high-order I.SUBm calculations for this model via a computational procedure for the Hamiltonian of $(7.43)$.

\subsubsection{CCM Results for the Ground-State Properties}

We note that for the CCM treatment of the interpolating Kagome/triangle model presented here (and see [65] for further details) the unit cell contains four lattice sites (see Fig. 7.13). By contrast. previous calculations [59] for the TAF used a unit cell containing only a single site per unit cell. Hence, the interpolating Kagomé/triangle model has many more "fundamental" configurations than the TAF model at equivalent levels of approximation. However. we find that those configurations which are not equivalent for the interpolating Kagome/triangle model but are equivalent for the TAF have CCM correlation coefficients $\left\{\mathcal{S}_{I}, \tilde{S}_{l}\right\}$ which become equal at the TAF point. $J^{\prime}=1$. Hence. the CCN naturally and without bias reflects the extra amomt of symmetry of the interpolating Kagome/triangle model at this one particular point. This is an excellent indicator of the validity of the CCM treatment of this model. The results for the interpolating Kagone/triangle model at $J^{\prime}=1$ thus also exactly agree with those of a previous CCM treatment of the TAF [59].

We now set $\lambda=1$ for the remainder of this subsection and again wo "track" the "trivial" solution for large $J^{\prime}$ for decreasing values of $J^{\prime}$ until we reach a critical value of $J^{\prime}$ at which the solution to the CCM equations breaks down. Results for $J_{2}^{\prime}$ for this model are presented in Table 7.2. A simple "heuristic" extrapolation of these results gives a value of $J_{c}^{\prime}=0.0 \pm 0.1$ for the position of this phase transition point. This result indicates that the classical three-sublattice Nelelike order. of which about $50 \%$ remains for the TAF. completely disappears at a point very near to the KAF point $\left(J^{\prime}=0\right)$.

The results for the ground-state energy are shown in Fig. 7.14 and in Table 7.2. These results are seen to be highly converged with respect to each other over the whole of the region $0 \leq J^{\prime} \leq 1$. The results for the groundstate energies of the KAF and TAF model in Table 7.2 agree well with results of other techniques. Indeed, we believe that the extrapolated CCM results 


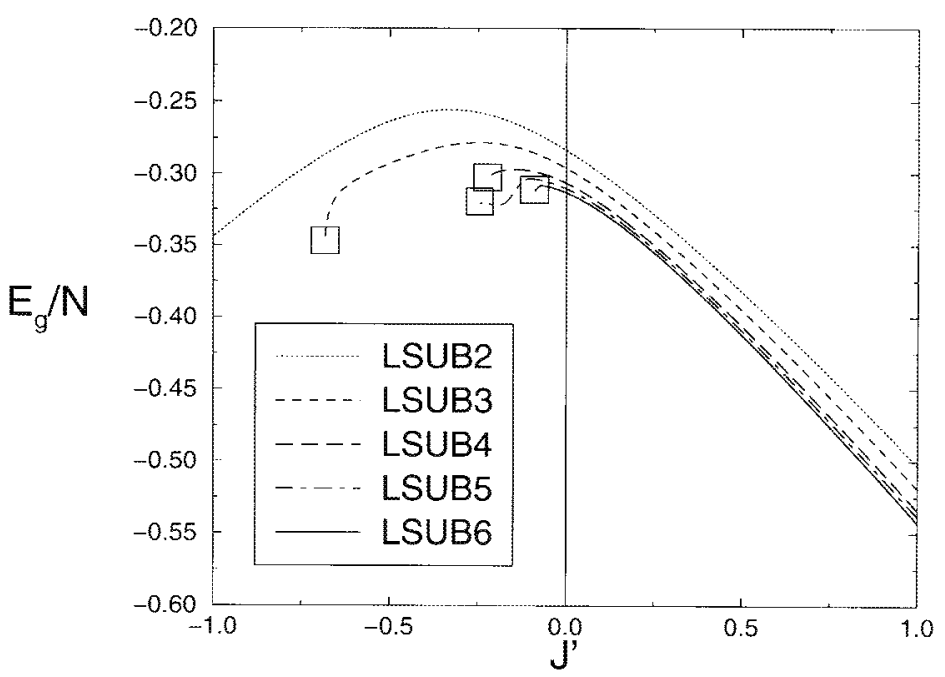

Fig. 7.14. CCM results for the ground-state energy per spin of the interpolating Kagomé/triangle model (with $J=1$ ) using the LSUBm approximation with $m=\{2,3,4,5,6\}$. The boxes indicate the CCM critical points, $J_{c}^{\prime}$, and a simple extrapolation in the limit $m \rightarrow \infty$ implies that $J_{c}^{\prime}=0.0 \pm 0.1$. (Figure taken from [65])

Table 7.2. CCM results [65] for the ground-state energy per spin and sublattice magnetisation of the TAF and KAF models using the LSUBm approximation with $m=\{2,3,4,5,6\}$. CCM critical values, $J_{c}^{\prime}$, of the interpolating Kagome/triang]e model (with $J=1$ ), which are themselves indicators of a phase transition point in the true system, are also given. Comparison is made in the last row with the results of other calculations

\begin{tabular}{|c|c|c|c|c|c|}
\hline & \multicolumn{2}{|c|}{ KAF } & \multicolumn{2}{c|}{ TAF } & $J-J^{\prime}$ \\
\hline$m$ & $E_{g} / N_{K}$ & $M^{K}$ & $E_{g} / N$ & $M^{K}$ & $J_{c}^{\prime}$ \\
\hline 2 & -0.37796 & 0.8065 & -0.50290 & 0.8578 & - \\
\hline 3 & -0.39470 & 0.7338 & -0.51911 & 0.8045 & -0.683 \\
\hline 4 & -0.40871 & 0.6415 & -0.53427 & 0.7273 & -0.217 \\
\hline 5 & -0.41392 & 0.5860 & -0.53869 & 0.6958 & -0.244 \\
\hline 6 & -0.41767 & 0.5504 & -0.54290 & 0.6561 & -0.088 \\
\hline$\infty$ & -0.4252 & 0.366 & -0.5505 & 0.516 & $0.0 \pm 0.1$ \\
\hline$c . f$. & $-0.43([89])$ & 0.0 & $-0.551([85])$ & $0.5([86,87])$ & - \\
\hline
\end{tabular}

are unquestionably among the most accurate results available for the groundstate energies of the TAF and KAF.

We now wish to describe how much of the original classical ordering of the model state remains for the quantum system. If one considers non-Kagomé 


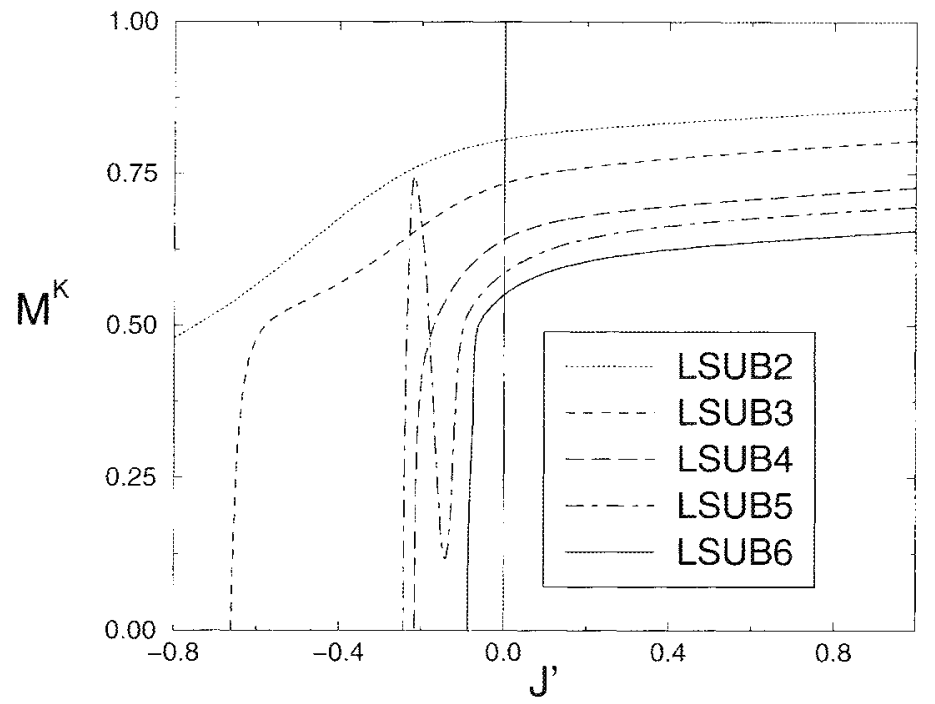

Fig. 7.15. CCM results for the sublattice magnetisation of the interpolating Kagomé/triangle model (with $J=1$ ) using the $\mathrm{LSUB} m$ approximation with $m=\{2,3,4,5.6\}$. (Figure taken from $[65]$ )

lattice sitcs then the spins on these sites are effectively "frozen" into their original directions (of the model state) at $J^{\prime}=0$. Hence, we believe that the relevant quantity to be considered for this model is the average value of $s_{k}^{z}$ (again after rotation of the local spin axes) where $k$ runs only over the $N_{K}$ Kagomé lattice sites, given by

$$
M^{K}=-\frac{2}{N_{K}} \sum_{k=1}^{N_{K}} s_{k}^{z} .
$$

The results for $M^{K}$ are presented in Fig. 7.15 and in Table 7.2. The puzzling "upturn" of $M^{K}$ for the LSUB5 data is an artifact, and typically such behaviour only ever occurs when one enters a phase in which the model state becomes an increasingly bad starting point. Although the extrapolated value for $M^{K}$ specifically at the KAF point remains non-zero, the LSUB6 result goes to zero very close to the KAF point. CCM results are thus fully consistent with the hypothesis that, unlike the TAF, the ground state of the KAF does not contain any Néel ordering.

\subsubsection{Evaluation of the Perturbation Series Using CCM}

Finally, it is instructive to make contact with the cumulant series expansions for the anisotropic TAF (i.e., $J^{\prime}=J=1$ ) with respect to the parameter $\lambda$. 
Table 7.3. Expansion coefficients in powers of $\lambda$ up to the 15 th order for the ground-state energy per spin, $E_{g} / N$, and the sublattice magnetisation, $M$, for the anisotropic spin- $\frac{1}{2}$ triangular-lattice Heisenberg antiferromagnet obtained from the CCM equations in the LSUB6 approximation. The exact series expansions up to the 11th order obtained by Singh and Huse [85] are also included for comparison. (Table taken from [59])

\begin{tabular}{|c|c|c|c|c|}
\hline Order & LSUB6: $E_{g} / \mathrm{N}$ & Exact: $_{g} / \mathrm{N}$ & LSUB6: $M$ & Exact: $M$ \\
\hline \hline 0 & -0.3750000 & -0.3750000 & 1 & 1 \\
\hline 1 & 0.0000000 & 0.0000000 & 0 & 0 \\
\hline 2 & -0.1687500 & -0.1687500 & -0.27 & -0.27 \\
\hline 3 & 0.0337500 & 0.0337500 & 0.108 & 0.108 \\
\hline 4 & -0.0443371 & -0.0443371 & -0.2726916 & -0.2726916 \\
\hline 5 & 0.0204259 & 0.0204259 & 0.1717951 & 0.1717951 \\
\hline 6 & -0.0283291 & -0.0283291 & -0.3315263 & -0.3315263 \\
\hline 7 & 0.0311703 & 0.0315349 & 0.4060277 & 0.4110737 \\
\hline 8 & -0.0357291 & -0.0476598 & -0.5331858 & -0.7382203 \\
\hline 9 & 0.0541263 & 0.0685087 & 0.8894023 & 1.1781303 \\
\hline 10 & -0.0771681 & -0.1025446 & -1.3927395 & -2.0109889 \\
\hline 11 & 0.1294578 & 0.1565522 & 2.4179612 & 3.4012839 \\
\hline 12 & -0.1848858 & $?$ & -4.0426184 & $?$ \\
\hline 13 & 0.2857225 & $?$ & 6.8086538 & $?$ \\
\hline 14 & -0.4463496 & $?$ & -11.488761 & $?$ \\
\hline 15 & 0.7021061 & $?$ & 19.388053 & $?$ \\
\hline
\end{tabular}

We have computed the perturbative CCM solutions of $E_{g} / N$ and the sublattice magnetisation $M$, as defined in (7.24) with respect to the local spin axes, in terms of the anisotropy parameter $\lambda$. In Table 7.3 we tabulate the expansion coefficients from the LSUB6 approximation, together with the corresponding results from cxact series expansions [85]. We note that the LSUB6 approximation reproduces the exact series expansion up to the sixth order. Wo conjecture that the LSUB $m$ approximation reproduces the exact series expansion to the same mth order. Moreover, the fact that the corresponding values of several of the higher-order expansion coefficients from both the CCM LSUB6 perturbative solution and the exact series expansion remain close to each other shows that the exponential parametrisation of the CCM with the inclusion of multi-spin correlations up to a certain order also captures the dominant contributions to correlations of a few higher orders in the series expansions.

\subsection{The $J_{1}-J_{2}$ Ferrimagnet}

We now briefly present results for another frustrated model in which we have both nearest- and next-nearest-neighbour antiforromagnetic bonds. (The in- 
terested reader is referred to [68.77] for more details.) The Hamiltonian for the square-lattice spin-half/spin-one $J_{1}, J_{2}$ ferrimagnet is given by

$$
I I=J_{1} \sum_{\langle i, j\rangle} \mathrm{s}_{i} \cdot \mathrm{s}_{j}+J_{2} \sum_{\{i, k\}} \mathrm{s}_{i} \cdot \mathrm{s}_{k} .
$$

where $(i, j)$ runs over all nearest-neighbour bonds on the square lattice. $\{i, k\}$ rums over all next-nearest-neighbour bonds. We assume that $f_{1}=1$ aud $J_{2}>0$ throughout this article and so this model is frustrated. Note that one sublattice (A) of the square lattice is populated entirely by spin-one spins $\left(s_{A}=1\right)$ and the other sublattice $(B)$ is populated entirely by spin-half spins $\left(s_{B}=1 / 2\right)$. This model is an extension of the well-known spin-half

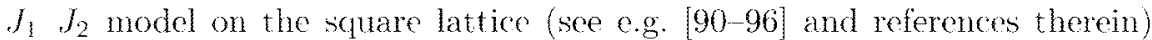
which serves as a canonical model for the discussion of an order-disorder quantum phase transition driven by the interplay of quantum fluctuations and frustration.

A feature of such ferrmagnetic spin systems is that the Lieb-Mattis theorem may be obeyed (if frustration is excluded) such that the gromd state has a magnetic moment per spin of strength $\left(s_{A}-s_{B}\right)=1 / 2$. Note in particular that this property is obeyed for the errimagnet of $(7.45)$ at $J_{2}=0$. and thus a maeroscopic lattice magnetisation exists for this case.

Wo note that many ferrimagnetic materials have recently been fabricated and various examples are the "ladder" systems: $\operatorname{InCu}(p b a \mathrm{OH})\left(\mathrm{H}_{2} \mathrm{O}\right)_{3}$ (where pba OH=2-hvdroxy-1. 3-propylenebisoxamato) and $\mathrm{AnCu}(p b a)\left(\mathrm{H}_{2} \mathrm{O}\right)_{3}$ - $\mathrm{H}_{2} \mathrm{O}$ (where $p b a=1.3$-propylenebisoxamato) $[9799]$. These materials contain magnetic atoms $\mathrm{Mn}\left(s_{A}=5 / 2\right)$ and $\mathrm{Cu}\left(s_{B}=1 / 2\right)$.

The classical behaviour of the square-lattice spin-half/spin-one ferrimagnet of (7.45) is also interesting and three distinct phases are predicted. The first such phase for $J_{2} \leq 0.25$ is one in which the ground-state is the collinear ferrimagnetic Néd state (shown in Fig. 7.16). A second-order phase transition then oceurs within this classical picture to a phase in which the spin-one spins may cant at an angle $\theta$. although the spin-half spins do not change their direction (also shown in Fig. 7.16). This state is henceforth referred to as the "spin-flop" state. A first-order phase transition to a collinear state in which next-nearest-neighbour spins are antiparallel (again. see Fig. 7.16) then occurs classically, and this state is roferred to here as the "collinear striped" state. Note that this state in the classical model is degenerate with states canting at an arbitrary angle between spins on sublattice A and spins on sublattice B. However. this degeneracy is lifted by quantum fuctuations which select the collinear state $[92.100 .101]$. We note that the spin-flop and collinear striped states are "incommensurate" in the sense that no value of the angle $\theta$ may be chosen such that the two states are equivalent. We note however that the Neel and spin-flop states are equivalent when $\theta=\pi$.

A further motivation for studying this model is that exact calculations of finite-sized lattices indicate that the behaviour of the quantmm ferrimagnet of 


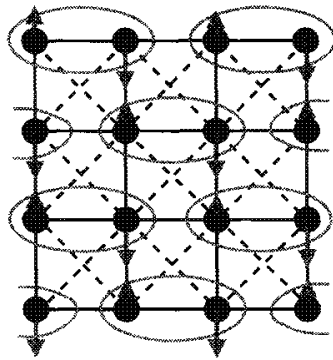

a

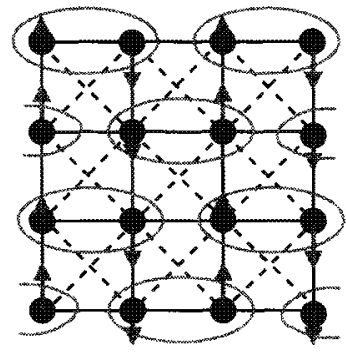

b

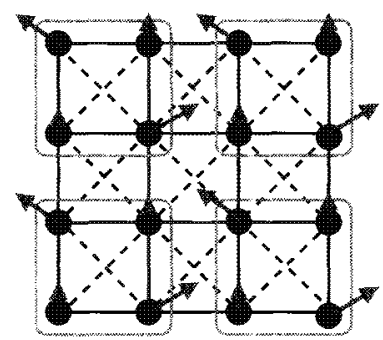

c

Fig. 7.16. The classical ground states used to treat the square-lattice spinhalf/spin-one $J_{1}-J_{2}$ ferrimagnet via the CCM. These states are nanely: a) the collinear ferrimagnetic Néel state; b) the collinear "striped" state; and c) the "spinfop" state. Note that long arrows indicate spin-one spins (and their orientation) and short arrows indicate the spin-half spins. The prinitive unit cells are shown by the ovals for the Neel and striped states and by the rectangles for the spin-flop state. Full lines indicate the nearest-neighbour bonds and dashed lines indicate the next-nearest-neighbour bonds. (Figure taken from [77])

(7.45) may be much different from the behaviour of its linear chain counterpart and from the square-lattice spin-half $J_{1}-J_{2}$ antiferromagnet. These exact calculations of finite-sized lattices suggest that the behaviour of the squarelattice ferrimagnet is analogous to that of the classical behaviour outlined above.

Three model states are used to treat the square-lattice spin-half/spinone $J_{1}-J_{2}$ ferrimagnet in order to provide results across various regimes of differing quantum order. The first such model state is the collinear Néel state, and the primitive unit cell in this case contains a spin-half site and a spin-one site (shown in Fig. 7.16). The underlying Bravais lattice is formed from two vectors $(\sqrt{2}, \sqrt{2})$ and $(\sqrt{2},-\sqrt{2})$. There is also an 8-point symmetry group, namely: rotations of $0^{\circ}, 90^{\circ}, 180^{\circ}$, and $270^{\circ}$; and reflections about the lines $x=0, y=0, y=x$, and $y=-x$. The collinear striped state (also shown in Fig. 7.16) is also used as a model state, in which spins for even values of $x$ along the $x$-axis point "downwards" and spins for odd values of $x$ point "upwards." The primitive unit cell again contains only two spins, although this time only four of the eight point group symmetry operations are allowed, namely: rotations of $0^{\circ}$ and $180^{\circ}$; and reflections about the lines $x=0$ and $y=0$. We note that rotations of the local axes of $180^{\circ}$ about the $y$-axis of cvery spin is carried out such that each spin now appears (mathematically) to point "downwards". Each spin may now be treated equivalently.

It is noted that (in the original unrotated spin coordinates) $s_{T}^{z} \equiv \sum_{i} s_{i}^{z}=$ 0 is preserved for all CCM approximations for these two models states in order to reduce the number of fundamental configurations at a given LSUBm or SUBm-m approximation level. 


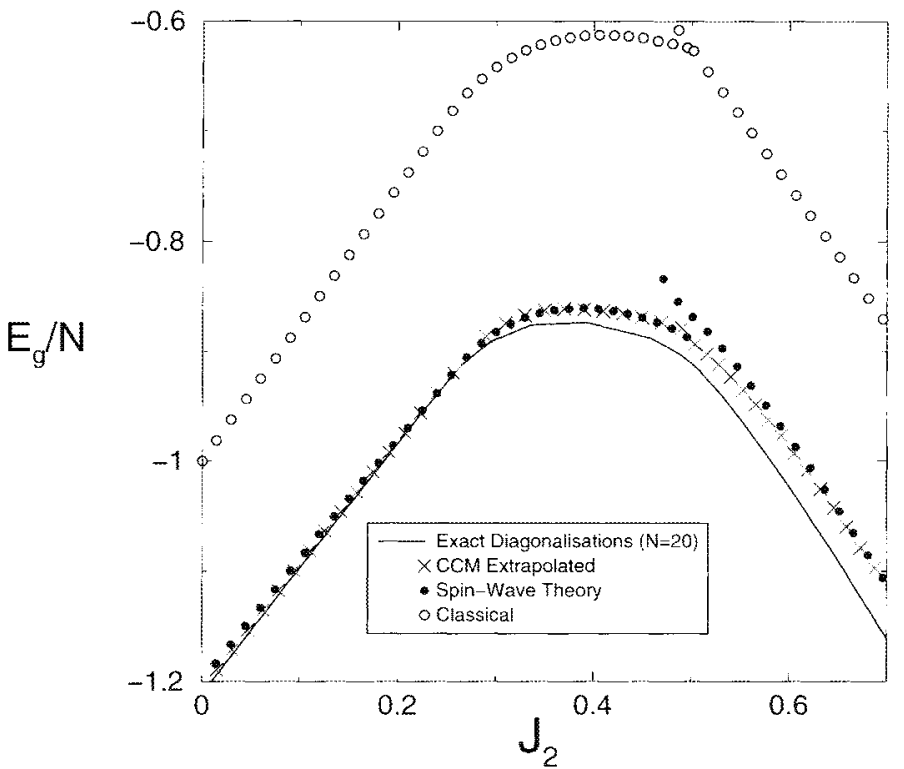

Fig. 7.17. Ground-state energy per spin of the square-lattice spin-half/spin-one $J_{1}$ $J_{2}$ ferrimagnet versus $J_{2}$ for the CCXI method using three model states compared to results of exact diagonalisations of finite-sized lattices. (Note that we have set $\left.I_{1}=1\right)$

The third model state is the spin-flop state. We note that there is no equivalent conserved quantity to siz. for the spin-flop model state, although single-body correlations are explicitly excluded from this calculation as they are (in some sense) already inchuded by the rotation of the local axes of spins. It should however be noted that this is an explicit assumption of the calculation for the spin-flop model state.

The amount of ordering on each sublattice is represented by.

$$
m_{1}=\frac{1}{N_{A}}\left|\sum_{i_{A}}^{N_{1}}\left\langle\tilde{\Psi}\left|s_{i_{A}}^{\tilde{z}}\right| \Psi\right\rangle\right|: m_{2}=\frac{1}{N_{B}}\left|\sum_{i_{H 3}}^{N_{I 3}}\left\langle\tilde{\Psi}\left|s_{i_{B}}^{\tilde{z}}\right| \Psi\right\rangle\right|
$$

where $i_{A}$ vuns over all spin-one lattice sites and $i_{B}$ runs over all spin-half lattice sites. Note that as usual, all of the spins for all of the models states have been rotated such that all spins appear mathematically to point downwards. The quantities $m_{1}$ and $m_{2}$ are the expectation values of the magnetic moment in the $z$-direction on the $A$ and $B$ sublattice, respectively, with respect to a given model state and represent order parameters for this model.

The ground-state energies predicted by the CCM using the three model states are shown in Fig. 7.17 and once again CCM results are in good agreement with results of exact diagonalisations (ED) of finite-sized lattices and spin-wave theory. Results for the sublattice magnet isations shown in Fig. 7.18 


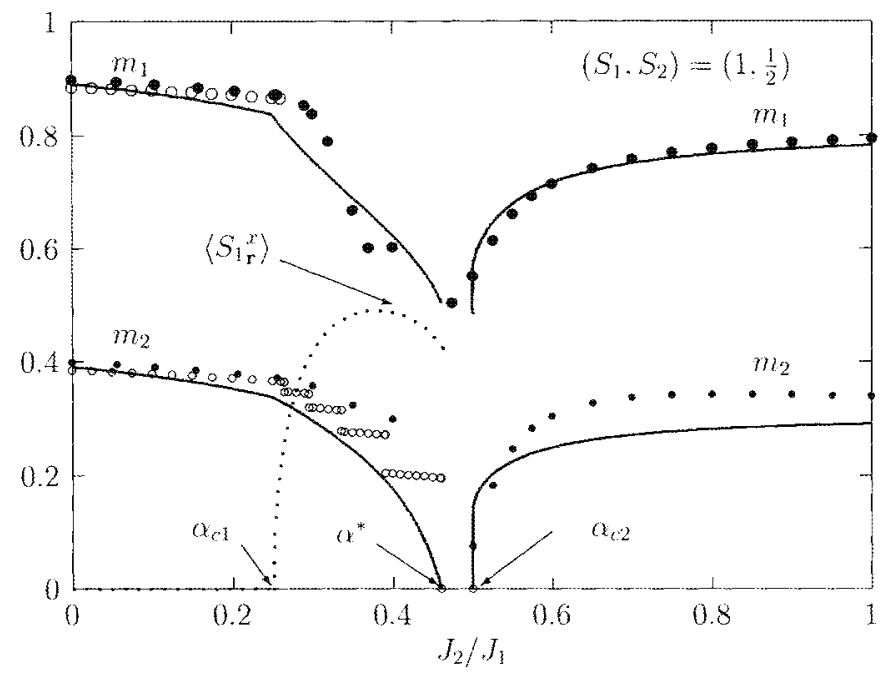

Fig. 7.18. Sublattice magnetisations, $m_{1}$ and $m_{2}$ of (7.46), of the square-lattice spin-half/spin-one $J_{1} \cdots J_{2}$ ferrimagnet versus $J_{2}$ for the CCM method using three model states compared to results of exact diagonalisations (ED) of finite-sized lattices. Note that full lines are those results for the sublattice magnetisation using linear spin-wave theory, open circles are those results of exact diagonalisations, and CCM results are indicated by the filled circles. (Figure taken from [68])

also show good correspondence with results of exact diagonalisations (ED) of finite-sized lattices and spin-wave theory. Hence, the CCM yields excellent quantitative accuracy for the ground-state properties of the spin-half/spinone $J_{1}-J_{2}$ ferrimagnet across a wide range of the next-nearest-neighbour bond strength $J_{2}$ by the use of three different model states. The CCM thus provides a comprehensive picture of the ordering in the ground state an accurate prediction of the phase diagram, and even evidence regarding the order of the phase transitions.

The results for the ground-state properties of the spin-half/spin-one $J_{1}$ $J_{2}$ ferrimagnet at $J_{1}=1$ and $J_{2}=0$ based on the Néel model state are presented in Table 7.4 and we see that our raw SUBm-m results appear to converge rapidly. An extrapolation in the limit $m \rightarrow \infty$ is also performed for the $J_{1} \cdot J_{2}$ ferrimagnet at $J_{1}=1$ and $J_{2}=0$ in order to provide even better accuracy.

The positions of quantum phase transition points as a function of $\alpha=$ $J_{2} / J_{1}$ are also shown in Fig. 7.18. A second-order phase transition is observed at $\alpha_{c_{1}}$ and CCM results place this at $\alpha_{c_{1}}=0.27$, which is slightly above the classical value. By contrast, CCM results predict a first-order phase transition at $\alpha_{\mathrm{C}_{2}} \approx 0.5$ and this result is in good agreement with those results of both spin-wave theory and exact diagonalisations. Another possible phase is also indicated in Fig. 7.18, namely, one is which we have finite and nonzero sublattice magnetisation on the spin-one lattice sites and zero sublattice 
Table 7.4. Results for the ground-state energy and amounts of sublattice magnetisations $m_{1}$ and $m_{2}$, on the spin-one and spin-half sites respectively. of the square-lattice spin-half/spin-one $J_{1} \quad J_{2}$ ferrmagnet at $J_{1}=1$ and $J_{2}=0$ based on the Néel model state [77]. Note that $N_{F}$ indicates the number of fundamontal configurations at a given level of $\mathrm{LSUB}$ or SUBm-m approximation. CCM results are compared to exact diagonalisations (ED) of finite-sized lattices. Heuristic extrapolations in the limit $m \rightarrow \infty$ are perfomed

\begin{tabular}{|c|c|c|c|c|}
\hline & $N_{F}$ & $E_{s} / N$ & $m_{1}$ & $m_{2}$ \\
\hline SUB2-2 & 1 & -1.192582 & 0.92848 & 0.42848 \\
\hline SUB4-4 & 13 & -1.204922 & 0.90781 & 0.40781 \\
\hline SUB6-6 & 268 & -1.206271 & 0.90333 & 0.40334 \\
\hline LSUB6 & 279 & -1.206281 & 0.90329 & 0.40330 \\
\hline Extrapolated CCN & & -1.2069 & 0.898 & 0.398 \\
\hline FD $(N=16)$ & & 1.218134 & 0.87515 & 0.37515 \\
\hline ED $(N=20)$ & & -1.212050 & 0.88482 & 0.38483 \\
\hline
\end{tabular}

magnetisation on the spin-half lattice sites. The onset of this phase with increasing $J_{2}$ is indicated by the symbol, $\alpha^{*}$.

\subsection{Conclusion}

We have seen in this chapter that the CCM may be applied to various quantum spin systems at zero temperature. In particular, suggestive results for the positions of CCM eritical points were observed, and these points were seen to correspond closely to the occurrence of quantum phase transitions in the "real" system. Furthermore, quantitatively accurate results for expectation values with respect to the ground and excited states wore determined. These results were seen to be competitive with the best results of other approximate methods.

A possible use of high-order CCN techniques might bo to predict excitation spectra of quant um magnets to great accuracy. Furthermore, this would mean that a direct comection might be made to results of neutron scattering experiments. Also, the application of the CCM to quantum spin systems which exhibit novel ordering. such as those with ground states which demonstrate dimer- or plaquette-solid ordering, is another possible future goal. Furthermore, the extension of high-order techniques to bosonic and fermionic systems is possible in future.

High-order CCM techniques might also be applied at even greater levels of approximation with the aid of parallel processing techniques [83]. Indeed the CCM is well-suited to such an implementation and recent CCM calculations using parallel processing techniques have been carried out for approximately $10^{4}$ fundamental configurations. We believe that an increase of at least ano- 
ther order of magnitude in the number of fundamental configurations should easily be possible in the near future by using such techniques.

The extension of the CCM to quantum spin systems at non-zero temperatures might also be accomplished by using thermo-field theory. The application of the CCM at both zero and non-zero temperatures might then help to explain the subtle interplay of quantum and thermal fuctuations in driving phase transitions over a wide range of physical parameters.

\section{References}

1. M.H. Kalos: Phys. Rev. 128, 1791 (1962).

2. D.M. Ceperley and M.H. Kalos. In: Monte Carlo Methods in Statistical Physics, edited by K. Binder (Springer Verlag, Berlin, 1979), p. 145.

3. K. Schmidt and M.H. Kalos. In: Applications of the Monte Carlo Method in Statistical Physics, edited by K. Binder (Springer Verlag, Berlin, 1984), p. 125.

4. R. Guardiola. In: Microscopic Quantum-Many-Body Theories and Their Applications, edited by J. Navarro and A. Polls, Lect. Notes Phys. 510, 269 (Springer-Verlag, Berlin-Heidelberg 1998).

5. J.W. Clark and E. Feenberg: Phys. Rev. 113, 388 (1959).

6. H.W. Jackson and E. Feenberg: Rev. Mod. Phys. 34, 686 (1962).

7. E. Feenberg. In: Theory of Quantum Liquids, edited by K. Binder, (SpringerVerlag, New York, 1969).

8. J.W. Clark. In: Progress in Particle and Nuclear Physics Vol. 2 , edited by D.H. Wilkinson, (Pergamon, Oxford, 1979), p. 89.

9. V.R. Pandharipande and R.B. Wiringa: Rev. Mod. Phys. 51, 821 (1979).

10. E. Krotscheck and J.W. Clark: Nucl. Phys. A333, 77 (1980).

11. J.W. Clark. In: The Many-Body Problem: Jastrow Correlations Versus Brueckner Theory, edited by R. Guardiola and J. Ros, Lect. Notes Phys. 138, 184 (Springer-Verlag, Berlin-Heidelberg 1981).

12. S. Rosati. In: International School of Physics Enrico Fermi, Course LXXIX, edited by A. Molinari (North-Holland, Amsterdam, 1981), p. 73.

13. A. Fabrocini and S. Fantoni. In: First International Course on Condensed Matter, edited by D. Prosperi, S. Rosati and S. Violini, ACIF Series, Vol. 8 (World Scientific, Singapore, 1987), p. 87.

14. S. Fantoni and V.R. Pandharipande: Phys. Rev. C 37, 1687 (1988).

15. S. Fantoni and A. Fabrocini. In: Microscopic Quantum Many-Body Theories and Their Applications, edited by J. Navarro and A. Polls, Lect. Notes Phys. 510, 119 (Springer-Verlag, Berlin-Heidelberg 1998).

16. F. Coester: Nucl. Phys. 7, 421 (1958): F. Coester and H. Kümmel, ibid. 17, 477 (1960).

17. J. Čizek: J. Chem. Phys. 45, 4256 (1966); Adv. Chem. Phys. 14, 35 (1969).

18. R.F. Bishop and K.H. Luhrmann: Phys. Rev. B 17, 3757 (1978).

19. H. Kümmel, K.H. Lührmann, and J.G. Zabolitzky: Phys Rep. 36C, 1 (1978).

20. J.S. Arponen: Ann. Phys. (N.Y.) 151, 311 (1983).

21. R.F. Bishop and H. Kümmel: Phys. Today 40(3), 52 (1987).

22. J.S. Arponen, R.F. Bishop, and E. Pajanne: Phys. Rev. A 36. 2519 (1987); ibid. 36, 2539 (1987); In: Condensed Matter Theories, Vol. 2, edited by P. Vashishta, R.K. Kalia, and R.F. Bishop (Plenum. New York, 1987), p. 357. 
23. R.J. Bartlett: J. Phys. Chem. 93, 1697 (1989).

24. R.F. Bishop: Theor. Chim. Acta 80, 95 (1991).

25. R. Jastrow: Phys. Rev. 98. 1479 (1955).

26. F. Iwamoto and M. Yamarla: Prog. Theor. Phys. 17. 543 (1957).

27. J.W. Clark and P. Westhaus: J. Math. Phys. 9, 131 (1968): P. Westhaus and J.W. Clark: ibid. 9. $149(1968)$.

28. M. Gaudin, J. Gillespie, and G. Ripka: Nucl. Phys. A176, 237 (1971); S. Fantoni and S. Rosati: Nuovo Cim. 20A, 179 (1974); G. Ripka: Phys. Rep. 56 1 (1979): Nuel. Phys. A314 115 (1979).

29. S. Fantoni and S. Rosati: Lett. Nuovo Cim. 10, 545 (1974); E. Krotscheck and M.L. Ristig: Phys. Lett. 48A. 17 (1974); Nucl. Phys. A242, 389 (1975).

30. S. Rosati and M. Viviani. In: First International Course on Condensed Matter. edited by D. Prosperi, S. Rosati, and G. Violini, ACIF Series, Vol. 8 (World Scientific. Singapore, 1988), p.231.

31. R.F. Bishop, D.J.J. Farmell, and J.B. Parkinson: Phys. Rev. B 61, 6775 (2000).

32. R.F. Bishop. D.J.I. Farnell, and Chen Zeng: Phys. Rev. B 59, 1000 (1999).

33. W. Marshall: Proc. R. Soc. London Ser. A 232, 48 (1955).

34. H. A. Bethe: Z. Phys. 71, 205 (1931).

35. L. Hulthén: Ark. Mat. Astron. Fys. A 26, No. 11 (1938).

36. R. Orbach: Plyss. Rev. 112, 309 (1958): C.N. Yang and C.P. Yang: ibid. 150. $321(1966) ;$ ibid. 150, $327(1966)$.

37. J. Des Cloiseanx and J.J. Pearson: Phys. Rev. 128, 2131 (1962); L.D. Faddeev and L.A. Takhtajan: Phys. Lett. 85A, 375 (1981).

38. S.R. White and R. Noack: Phys. Rev. Lett. 68, 3487 (1992): S.R. White: ibid. 69. 2863 (1992): Phys. Rev. B 4810345 (1993).

39. Lou-Dimensional Quantum Field Theories for Condensed Matter Physicists. edited by L. Yu. S. Lundquist, and G. Morandi, Series in Modern Condensed Matter Physies. Vol. 7 (World Scientific, Singapore, 1995).

40. M.L. Ristig and J.W. Kim: Phys. Rev, B 53. 6665 (1996).

41. M.L. Ristig. J.W. Kim. and J.W. Clark. In: Theory of Spin Lattices and Lattice Gauge Models, edited by J.W. Clark and M.L. Ristig. Lecture Notes in Physics, Vol. 494 (Springer-Verlag. Borlin 1997). p. 62.

42. M.L. Ristig. J.W. Kim and J.W. Clark: Phys. Rev. B 57, 56 (1998).

43. R.F. Bishop, D.J.J. Famell, and M.L. Ristig. In: Condensed Matter Theories. Vol. 14, edited by D.J. Frnst. I.E. Perakis, and A.S. Umar (Nova Science Publ. Huntington. New York, 2000). p. 191.

44. D. J. J. Farnell and M. L. Ristig. In: Advances in Quantum Many-Body Theory. Vol. 5. edited by Raymond F. Bishop. Klaus A. Gernoth. and Niels R. Walet. p. 223 (World Scientific, Singapore, 2001).

45. A.D. Jackson. A. Lande, and R.A. Smith: Phys. Rev. Lett. 54, 1469 (1985): E. Krotscheck, R.A. Smith. and A.D. Jackson: Phys. Rev. A 33, 3535 (1986).

46. R.F. Bishop. In: Recent Progress in Many-Body Theories. Vol. 4. edited by E. Schachinger. H. Mitter, and H. Sormann (Plemm Press, New York. 1995). p. 195 .

47. M. Roger and J.H. Wetherington: Phys. Rev. B 41. 200 (1990): Europhys. Lett. 11, $255(1990)$.

48. R.F. Bishop. J.B. Parkinson, and Y. Xian: Phys. Rev, B 44. 9425 (1991).

49. R.F. Bishop. J.B. Parkinson. and Y. Xian: Phys. Rev. B 46, 880 (1992).

50. R.F. Bishop, J.B. Parkinson, and Y. Xian: J. Phys.: Condens. Matter 5. 9169 (1993). 
51. D.J.J. Farnell and J.B. Parkinson: J. Phys.: Condens. Matter 6. 5521 (1994).

52. Y. Xian: J. Phys.: Condens. Matter 6, 5965 (1994).

53. R. Bursill, G.A. Gehring, D.J.J. Farnell, J.B. Parkinson, T. Xiang, and C. Zeng: J. Phys.: Condens. Matter 7, 8605 (1995).

54. R. Hale. Ph.D. Thesis, UMIST, Manchester, United Kingdom (1995).

55. R.F. Bishop, R.G. Hale, and Y. Xian: Phys. Rev. Lett. 73, 3157 (1994).

56. R.F. Bishop, D.J.J. Farnell, and J.B. Parkinson: J. Phys.: Condens. Matter 8, $11153(1996)$.

57. D.J.J. Farnell. S.A. Krüger, and J.B. Parkinson: J. Phys.: Condens. Matter 9. 7601 (1997).

58. R.F. Bishop, Y. Xian, and C. Zeng. In: Condensed Matter Theories, Vol. 11, edited by E.V. Ludeña, P. Vashishta, and R.F. Bishop (Nova Science, Commack. New York, 1996), p. 91.

59. C. Zeng, D.J.J. Farnell, and R.F. Bishop: J. Stat. Phys., 90, 327 (1998).

60. R.F. Bishop, D.J.J. Farnell, and J.B. Parkinson: Phys. Rev. B 58. 6394 (1998).

61. J. Rosenfeld. N.E. Ligterink, and R.F. Bishop: Phys. Rev. B 60. 4030 (1999).

62. R.F. Bishop, D.J.J. Farnell, S.E. Krüger, J.B. Parkinson, J. Richter, and C. Zeng: J. Phys.: Condens. Matter 12, 7601 (2000).

63. R.F. Bishop, D.J.J. Farnell, and M.L. Ristig: Int. J. Mod. Phys. B 14, 1517 (2000).

64. S.E. Krüger, J. Richter, J. Schulenberg, D.J.J. Farnell, and R.F. Bishop: Phys. Rev. B 61, 14607 (2000).

65. D.J.J. Famell, R.F. Bishop. and K.A. Gemoth: Phys. Rev. B 63, 220402R (2001).

66. D.J.J. Farnell, K.A. Gernoth, and R.F. Bishop: Phys. Rev. B 64, 172409 (2001).

67. D.J.J. Farnell, K.A. Gernoth, and R.F. Bishop: J. Stat. Phys. 108, 401 (2002).

68. N.B. Ivanov, J. Richter, and D.J.J. Famell: Phys. Rev. B 66, 014421 (2002).

69. C.H. Llewellyn Smith and N.J. Watson: Phys. Lett. B 302, 463 (1993).

70. R.F. Bishop, A.S. Kendall, L.Y. Wong, and Y. Xian: Phys. Rev. D 48, 887 (1993): S.J. Baker, R.F. Bishop, and N.J. Davidson: Phys. Rev. D 53. 2610 (1996): Nucl. Phys. B (Proc. Supp.) 53, 834 (1997).

71. N.E. Ligterink. N.R. Walet, and R.F. Bishop: Ann. Phys. (NY) 284, 215 (2000).

72. N.E. Ligterink, N.R. Walet, and R.F. Bishop: Ann. Phys. (NY) 267, 97 (1998); Phys. Rev. E 63, 037103 (2001).

73. T. Barnes, D. Kotchan, and E.S. Swanson: Phys. Rev. B 39, 4357 (1989).

74. P. W. Anderson: Phys. Rev. 86, 694 (1952); T. Oguchi: Phys. Rev. 117, 117 (1960).

75. W. Zheng; J. Oitmaa, and C.J. Hamer: Phys. Rev. B 43, 8321 (1991).

76. A.W. Sandvik: Phys. Rev. B 56, 11678 (1997).

77. S.E. Krüger, D.J.J. Farnell, and J. Richter. In: Proceedings of the $11^{\text {th }}$ International Conference on Recent Progress in Many-Body Theories, edited by R.F. Bishop, T. Brandes, K.A. Gernoth, N.R. Walet, and Y. Xian, Series on Advances in Quantum Many-Body Theory Vol. 6, (World Scientific, Singapore, 2002), p. 365.

78. M. Suzuki: J. Phys. Soc. Japan 55, 4205 (1986).

79. R.R.P. Singh. M.P. Gelfand, and D.A. Huse: Phys. Rev. Lett. 61 2484, (1988).

80. N.B. Ivanov, S.E. Krüger, and J. Richter: Phys. Rev. B 53 2633. (1996). 
81. S.E. Krïger and J. Richter: Phys. Rev. B 64024433, (2001).

82. P. Tomczak and J. Richter: J. Phys. A: Math. Gen. 34 L461, (2001).

83. D.J.J. Farnell and R.F. Bishop: arXiv:cond-mat/0311126.

84. S. Chakravarty, B.I. Halperin and D.R.Nelson: Phys. Rev. B 39 2344, (1989).

85. R.R.P. Singh and D.A. Huse: Phys. Rev. Lett. 68, 1766 (1992).

86. B. Bernu, P. Lecheminant, C. Lhuillier, and L. Pierre: Phys. Scripta T49. 192 (1993); Phys. Rev. B 50. 10048 (1994).

87. T. Jolicoeur and J.C. LeGuilloul: Phys. Rev. B 40, 2727 (1989).

88. P. Ledheminant. B. Bermu. C. Lhuillier. T. Pierre and P. Sindzingre: Phys. Rev. B 56, 2521 (1997).

89. C. Waldtmann. H.U. Everts, B. Bernu. C. Lhuiller. P. Sindzingre. P. Lecheminant. and L. Pierre: Eur. Phys. J. B 2. 501 (1998).

90. P. Chandra and B. Docont: Phys. Rev. B 38, 9335 (1988).

91. H.J. Schulz and T.A.L. Ziman: Europhys. Lett. 18, 355 (1992); H.J. Schulz. T.A.L. Ziman. and D. Poilblanc: J. Phys. I 6, 675 (1996).

92. J. Richter: Plys. Rev. B 47. 5794 (1993).

93. J. Oitmaa and Zheng Weihong: Phys. Rev. B 54, 3022 (1996).

94. R.R.P. Singh. Zheng Weihong. C.J. Hamer, and J. Oitmaa: Phys. Rev. B 60. $7278(1999)$.

95. V.N. Kotor and O.P. Sushkov: Phys. Rev. B 61. 11820 (2000).

96. L. Capriotti and S. Sorella: Phys. Rev. Lett. 84. 3173 (2000).

97. O. Kahn. Y. Pei. and Y. Jornauz. In: Inorganic Materials edited by D.W. Bruce and D.O. OHare (John Wiley \& Sons Ltd. New York. 1992).

98. N.B. Ivanov, J. Richter, and U. Schollwöck: Phys. Rev. B 58, 14456 (1998).

99. J. Richter. U. Schollwöck. and N. B. Ivanov: Physica B $281 \& 282845$ (2000).

100. A. Moreo. F. Dagotto. T. Jolicoeur and J. Riera: Phys. Rev. B 42.6283 (1990).

101. K. Kubo and T. Kishi: J. Phys. Soc. Jpn. 60. 567 (1991). 